\title{
A very efficient Phosphoramidite Ligand for Asymmetric Iridium-Catalyzed Allylic Alkylation
}

\author{
Alexandre Alexakis* and Damien Polet \\ Department of Organic Chemistry, University of Geneva, 30, quai Ernest Ansermet, CH-1211 \\ Geneva 4, Switzerland
}

Supporting Information

General Procedures. ${ }^{1} \mathrm{H}(400 \mathrm{MHz})$ and ${ }^{13} \mathrm{C}(100 \mathrm{MHz}) \mathrm{NMR}$ spectra were recorded in $\mathrm{CDCl}_{3}$, and chemical shift $(\delta)$ are given in ppm relative to residual $\mathrm{CHCl}_{3}$. Evolution of reaction was followed by GC-MS Hewlett Packard (EI mode) HP6890-5973. Optical rotations were measured at $22^{\circ} \mathrm{C}$ in a $10 \mathrm{~cm}$ cell in the stated solvent; $[\alpha]_{D}$ values are given in $10^{-1}$ deg. $\mathrm{cm}^{2} \mathrm{~g}^{-1}$ (concentration c given as g/100 mL). Enantiomeric excesses were determined by chiral-GC (capillary column, $10 \mathrm{psi} \mathrm{H}_{2}$ ) or chiral-SFC with the stated column. Temperature programs are described as follows: initial temperature $\left({ }^{\circ} \mathrm{C}\right)$ - initial time (min) temperature gradient $\left({ }^{\circ} \mathrm{C} / \mathrm{min}\right)$ - final temperature $\left({ }^{\circ} \mathrm{C}\right)$; retention times $\left(\mathrm{R}_{\mathrm{T}}\right)$ are given in min. Flash chromatography was performed using silica gel 32-63 $\mu \mathrm{m}, 60 \AA . \mathrm{CH}_{2} \mathrm{Cl}_{2}$, was distilled from $\mathrm{CaH}_{2}$ under nitrogen. THF was dried by passage over alumina (activated at $350{ }^{\circ} \mathrm{C}$ under nitrogen atmosphere for $12 \mathrm{~h}$ ) .

Substrates were synthesized by known experimental procedures. ${ }^{1}$

Lithium chloride was dried over 24 hours at $80^{\circ} \mathrm{C}$ prior to use.

$[\operatorname{Ir}(\mathrm{COD}) \mathrm{Cl}]_{2}$ was purchased from Strem and used as received.

Ligands $\mathbf{L 1} \mathbf{b}-\mathbf{L} 3 \mathbf{b}$ were prepared according to the reported procedure. ${ }^{2}$

The sodium malonate solution was prepared as followed : $\mathrm{NaH}$ (dispersion on mineral oil, 50 $\%, 0.049 \mathrm{~g}, 1 \mathrm{mmol}$ ) was rinsed 3 times with portions of $n$-pentane $(5 \mathrm{~mL})$, one portion of dry THF (5 mL) and then suspended in $1.5 \mathrm{~mL}$ of dry THF. Dimethylmalonate $(0.115 \mathrm{~mL}, 1$ mmol) was added dropwise under stirring at room temperature. The resulting solution was immediately used.

\section{General procedure for the Ir-catalyzed allylic alkylation.}

In a flame-dried Schlenk tube, $\mathbf{L} 1(0.022 \mathrm{mmol})$ and $\operatorname{Ir}(\mathrm{COD}) \mathrm{Cl}$ dimer $(0.0067 \mathrm{~g}, 0.01 \mathrm{mmol})$ were dissolved in $0.5 \mathrm{~mL}$ of THF. The resulting orange solution was stirred for 20 minutes at room temperature and treated with the substrate $(0.5 \mathrm{mmol})$ as well as a freshly prepared sodium malonate solution ( $1 \mathrm{mmol}$ in $1.5 \mathrm{~mL}$ THF). The reaction mixture was stirred at the indicated temperature until complete disappearance of the starting material. The mixture was hydrolyzed with water, extracted with diethyl ether and dried over magnesium sulfate. The product was concentrated in vacuo, then $5 \mathrm{~mL}$ of pentane were added to let the catalyst sink. The resulting suspension is filtered, concentrated in vacuo and chromatographied on silica gel (n-pentane/diethyl ether 7:3) to afford the adduct as a colorless oil.

\footnotetext{
${ }^{1}$ Lehmann, J.; Lloyd-Jones, G. C. Tetrahedron 1995, 51, 8863-8874.

${ }^{2}$ Alexakis, A.; Tissot-Croset, K.; Polet, D. Angew. Chem. Int. Ed. 2004, 43, 2426-2428.
} 
<smiles>C=CC(C(=O)OC)C(C(=O)OC)c1ccccc1</smiles>

(R)-2-(1-Phenyl-allyl)-malonic acid dimethyl ester $\mathbf{2 a}^{\mathbf{1}} \mathrm{H}$ NMR (400.13 $\left.\mathrm{MHz}, \mathrm{CDCl}_{3}\right) 7.40-7.23$ (m, 5H), 6.03 (ddd, $1 \mathrm{H}, J=18.2,10.1,8.1 \mathrm{~Hz}$ ), $5.15(\mathrm{~d}, 1 \mathrm{H}, J=18.0 \mathrm{~Hz}), 5.12$ (d, $1 \mathrm{H}, J=10.6 \mathrm{~Hz}), 4.15$ (dd, $1 \mathrm{H}, J=$ 10.8, 8.1 Hz), 3.90 (d, $1 \mathrm{H}, \mathrm{J}=11.1 \mathrm{~Hz}), 3.78(\mathrm{~s}, 3 \mathrm{H}), 3.53(\mathrm{~s}, 3 \mathrm{H}) .{ }^{13} \mathrm{C}$ NMR (100.59 MHz, $\left.\mathrm{CDCl}_{3}\right)$ 168.2, 167.8, 139.9, 137.8, 128.7, 127.9, 127.2, 116.7, 57.4, 52.6, 52.4, 49.8. MS (EI) 189 (98), 157 (19), 129 (43), 117 (100), 91 (17), 77 (10). $[\alpha]_{D}^{22}=+34.1$ (c 1.08, $\mathrm{CHCl}_{3}$ ) for $98 \%$ ee. determined by comparison with published data. ${ }^{3}$ Ee was measured by chiral SFC analysis with a Regis (R,R) WHELK-O column ( $2 \% \mathrm{MeOH}$ during $2 \mathrm{~min}$, then $1 \% \mathrm{~min}^{-1}$, flow rate $2 \mathrm{~mL} / \mathrm{min}$, pressure 130 bars, $\left.0^{\circ} \mathrm{C}\right) \mathrm{R}_{\mathrm{T}}: 3.05(R), 3.29(S)$.
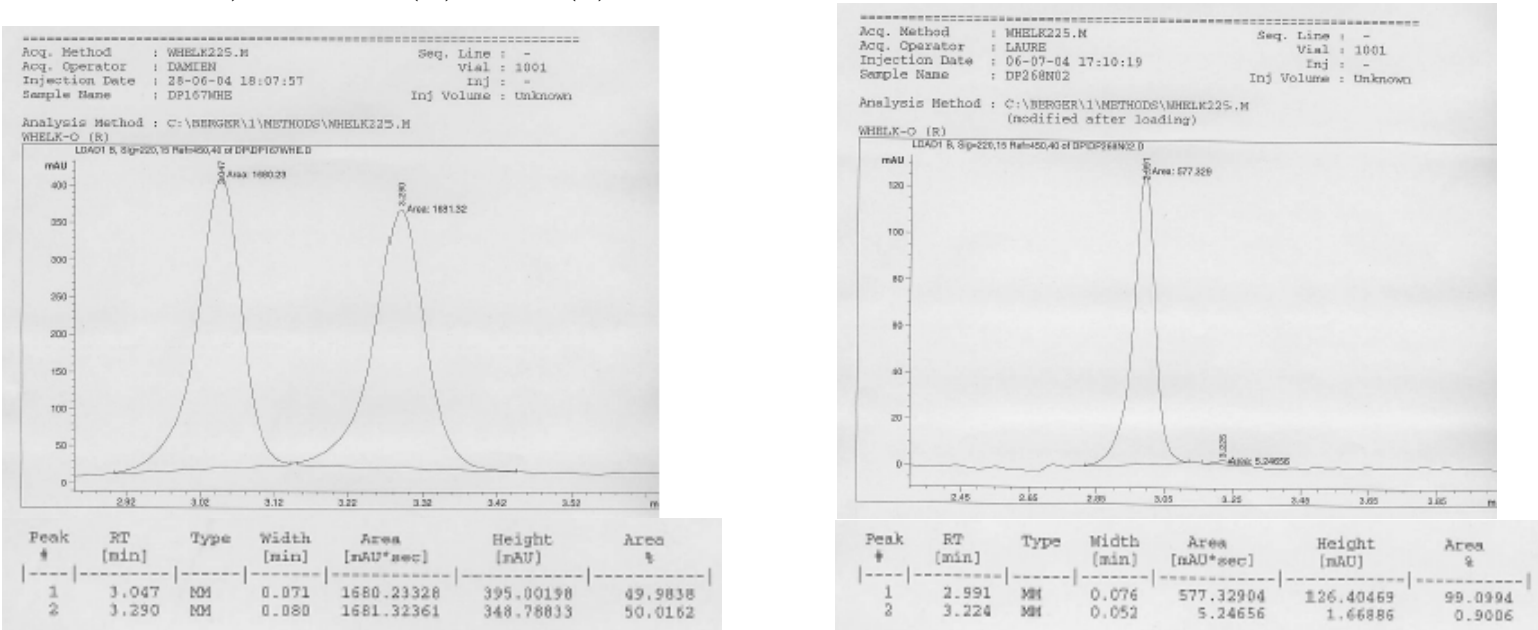<smiles>C=CC(C(=O)OC)C(C(=O)OC)c1ccc2ccccc2c1</smiles>

(+)-2-(1-Naphthalen-2-yl-allyl)-malonic acid dimethyl ester $2 \mathbf{b b}^{4}{ }^{\mathbf{1}} \mathrm{H}$ NMR (400.13 MHz, $\left.\mathrm{CDCl}_{3}\right)$ 7.81-7.79 (m, 3H), 7.69 (s, 1H), 7.477.44 (m, 2H), 7.37 (dd, $1 \mathrm{H}, J=8.6,1.5 \mathrm{~Hz}$ ), 6.08 (ddd, $1 \mathrm{H}, J=17.2$, 10.4, $8.1 \mathrm{~Hz}$ ), 5.18 (d, $1 \mathrm{H}, J=17.2 \mathrm{~Hz}$ ), 5.04 (d, $1 \mathrm{H}, J=10.1 \mathrm{~Hz}$ ), 4.30 (dd, $1 \mathrm{H}, J=9.6,8.4 \mathrm{~Hz}$ ), 4.01 (d, $1 \mathrm{H}, J=11.1 \mathrm{~Hz}$ ), 3.77 (s, 3H), 3.46 (s, 3H). ${ }^{13} \mathrm{C}$ NMR (100.59 $\left.\mathrm{MHz}, \mathrm{CDCl}_{3}\right)$ 168.3, 167.9, 137.7, 137.5, 133.5, 132.6, 128.4, 127.9, 127.7, 126.7, 126.2, 126.1, 125.9, 116.9, 57.3, 52.7, 52.5,

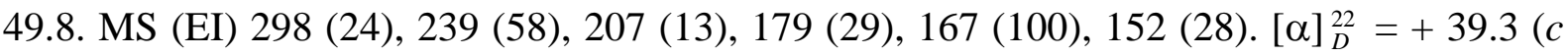
$0.925, \mathrm{CHCl}_{3}$ ) for $96 \%$ ee. Ee was measured by chiral SFC analysis with a Chiralcel OB-H column ( $2 \% \mathrm{MeOH}$ during $2 \mathrm{~min}$, then $1 \% \mathrm{~min}^{-1}$, flow rate $2 \mathrm{~mL} / \mathrm{min}$, pressure 200 bars, $\left.30^{\circ} \mathrm{C}\right) \mathrm{R}_{\mathrm{T}}: 7.43(+), 8.05(-)$.
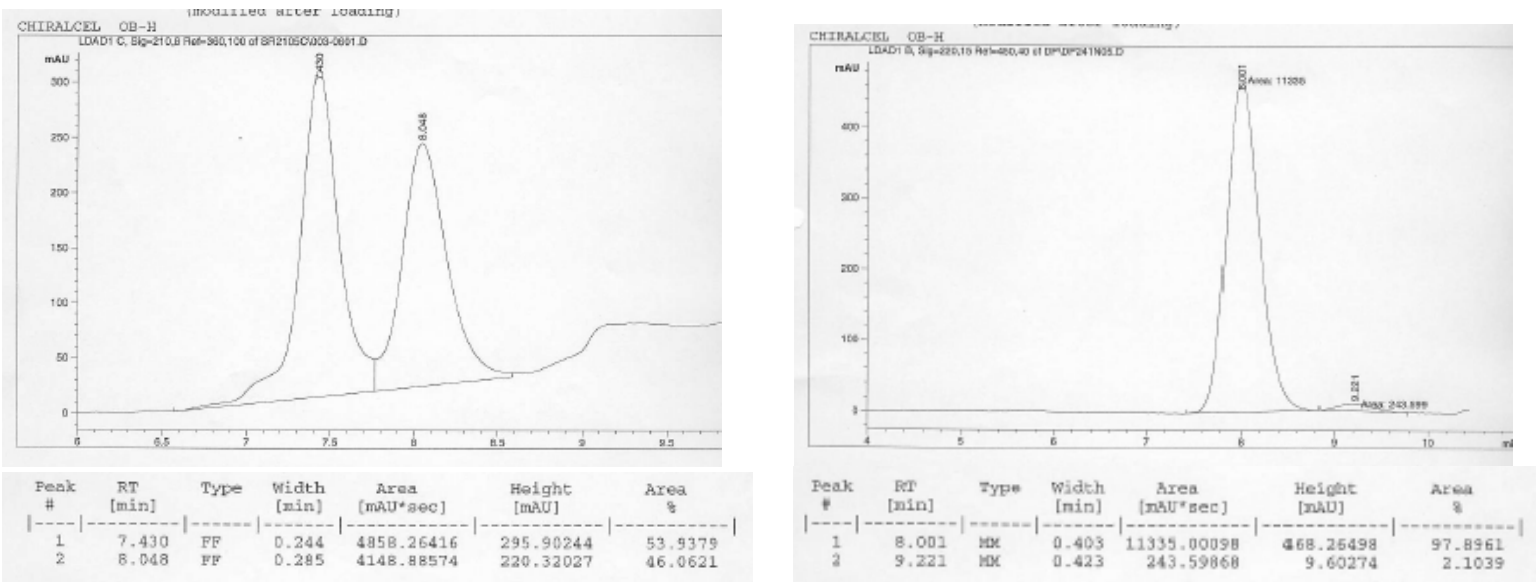

\footnotetext{
${ }^{3}$ Lloyd-Jones, G. C.; Pfaltz, A. Angew. Chem. Int. Ed. 1995, 34, 462-464.

${ }^{4}$ Pretot, R.; Pfaltz, A. Angew. Chem. Int. Ed. 1998, 37, 323-325.
} 
<smiles>C=CC(C(=O)OC)C(C(=O)OC)c1ccc2c(c1)OCO2</smiles>

(+)-2-(1-Benzo[1,3]dioxol-5-yl-allyl)-malonic acid dimethyl ester 2c ${ }^{1} \mathrm{H}$ NMR (400.13 MHz, $\left.\mathrm{CDCl}_{3}\right)$ 7.74-7.66 (m, 3H), 5.98-5.89 (m, 3H), 5.10 (d, 1H, $J=15.4 \mathrm{~Hz}$ ), 5.07 (d, 1H, $J=8.8 \mathrm{~Hz}$ ), 4.03 (dd, 1H, $J=$ 10.9, $8.1 \mathrm{~Hz}), 3.79$ (d, $1 \mathrm{H}, J=10.9 \mathrm{~Hz}), 3.73(\mathrm{~s}, 3 \mathrm{H}), 3.54(\mathrm{~s}, 3 \mathrm{H}) .{ }^{13} \mathrm{C}$ NMR (100.59 MHz, $\left.\mathrm{CDCl}_{3}\right)$ 168.3, 167.9, 139.9, 137.7, 137.5, 133.5, 132.6, 128.4, 127.9, 127.7, 126.7, 126.2, 126.1, 125.9, 116.9, 57.3, 52.7, 52.6, 52.5, 49.8. MS (EI) 298 (24), 239 (58), 207 (13), 179 (29), 167 (100), 152 (28).

HRMS (EI) calcd for $[\mathrm{M}+\mathrm{Na}]^{+} 315.0845$, found 315.0834. $[\alpha]_{D}^{22}=+22.0\left(c\right.$ 1.13, $\left.\mathrm{CHCl}_{3}\right)$ for $97 \%$ ee. Ee was measured by chiral SFC analysis with a Regis $(\mathrm{R}, \mathrm{R})$ WHELK-O column $(1 \%$ $\mathrm{MeOH}$ during $6 \mathrm{~min}$, then $1 \% \mathrm{~min}^{-1}$, flow rate $2 \mathrm{~mL} / \mathrm{min}$, pressure $130 \mathrm{bars}, 30^{\circ} \mathrm{C}$ ) $\mathrm{R}_{\mathrm{T}}: 6.02$ $(+), 6.77(-)$.
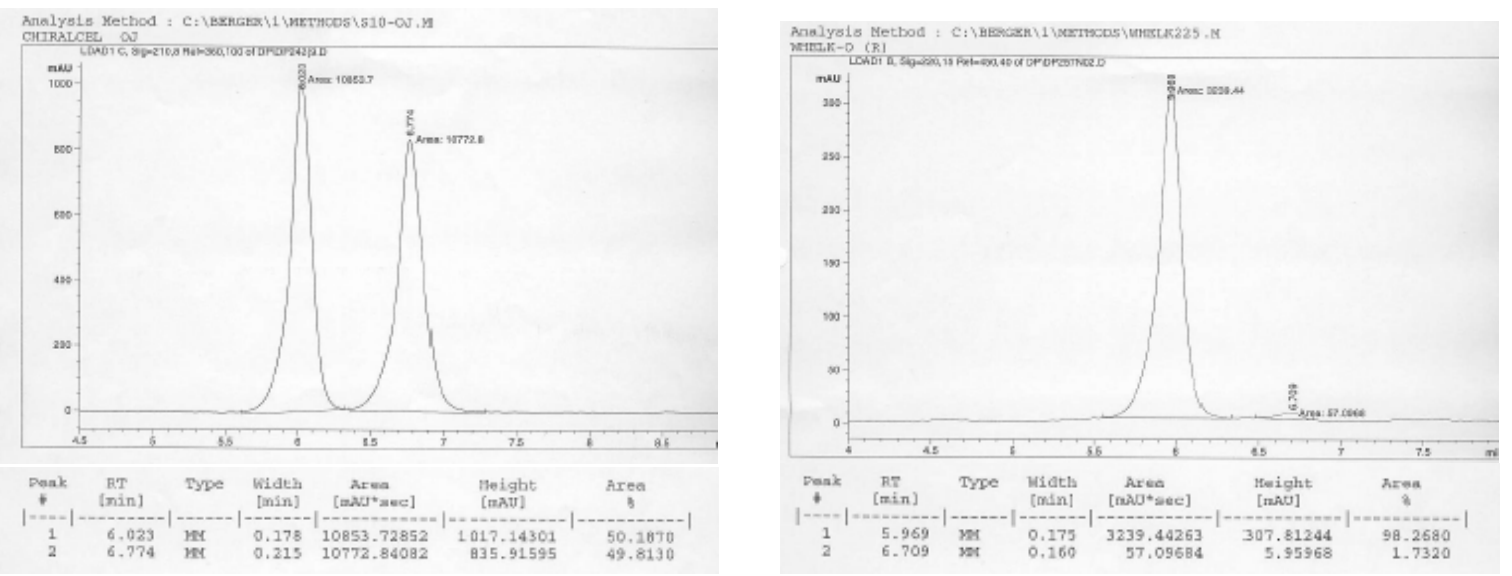<smiles>C=C[C@H](c1ccc(C(F)(F)F)cc1)C(C(=O)OC)C(=O)OC</smiles>

(+)-2-[1-(4-Trifluoromethyl-phenyl)-allyl]-malonic acid dimethyl ester $2{ }^{1} \mathrm{H}$ NMR (400.13 $\left.\mathrm{MHz}^{1} \mathrm{CDCl}_{3}\right) 7.57(\mathrm{~d}, 2 \mathrm{H}, J=8.1 \mathrm{~Hz})$, 7.36 (d, $2 \mathrm{H}, J=8.3 \mathrm{~Hz}$ ), 5.97 (ddd, $1 \mathrm{H}, J=16.9,10.1,8.1 \mathrm{~Hz}$ ), 5.16 (d, $1 \mathrm{H}, J=7.6 \mathrm{~Hz}$ ), 5.12 (s, 1H), 4.19 (dd, $1 \mathrm{H}, J=10.9,8.6 \mathrm{~Hz}$ ), 3.90 $(\mathrm{d}, 1 \mathrm{H}, \mathrm{J}=11.1 \mathrm{~Hz}), 3.76$ (s, 3H), 3.53 (s, 3H). ${ }^{13} \mathrm{C}$ NMR (100.59 $\left.\mathrm{F}_{3} \mathrm{C} \quad \mathrm{MHz}_{\mathrm{CDCl}}\right)$ 167.8, 167.5, 144.1, 136.9, 129.6, 129.2, 128.3, 125.6125.5 (q, 1C, $J=3.3 \mathrm{~Hz}$ ), 125.4, 117.5, 56.9, 52.7, 52.5, 49.3. MS (EI) 260 (10), 257 (100),

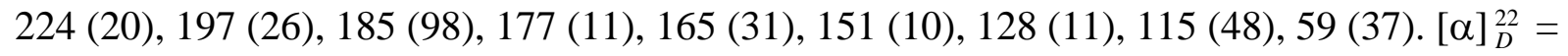
+40.1 ( $c 0.81, \mathrm{CHCl}_{3}$ ) for $94 \%$ ee. Ee was measured by chiral GC analysis with a Chiraldex LIPODEX E column (60-0-1-170-5) $\mathrm{R}_{\mathrm{T}}: 59.09(-), 59.68$ (+).
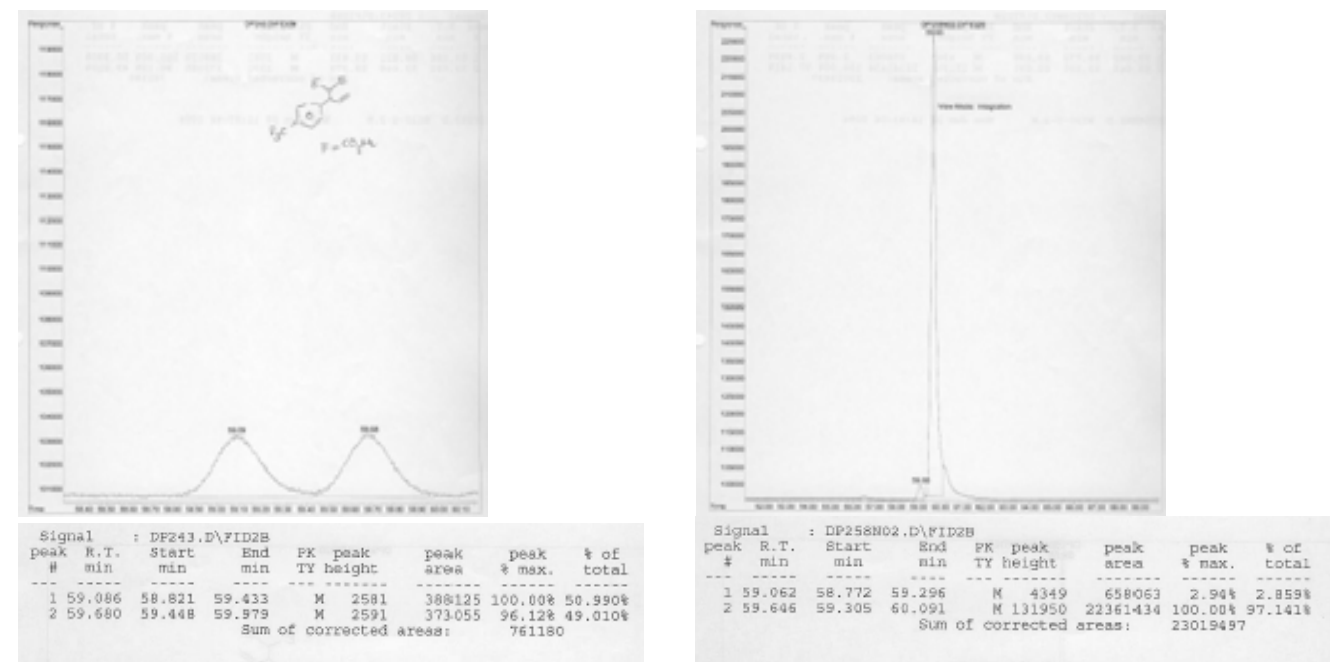
<smiles>C=CC(C(=O)OC)C(C(=O)OC)c1ccc(Cl)cc1</smiles>

\section{(+)-2-[1-(4-Chloro-phenyl)-allyl]-malonic acid dimethyl ester $2 e$} ${ }^{1} \mathrm{H}$ NMR (400.13 MHz, $\left.\mathrm{CDCl}_{3}\right) 7.30$ (d, 2H, $J=8.3 \mathrm{~Hz}$ ), 7.20 (d, 2H, $J$ $=8.3 \mathrm{~Hz}$ ), 5.99 (ddd, $1 \mathrm{H}, J=16.9,10.4,8.1 \mathrm{~Hz}$ ), 5.16 (d, 1H, $J=7.1$ $\mathrm{Hz}$ ), 5.12 (d, 1H, $J=0.8 \mathrm{~Hz}$ ), 4.13 (dd, $1 \mathrm{H}, J=10.9,8.1 \mathrm{~Hz}$ ), 3.85 (d, $1 \mathrm{H}, \mathrm{J}=10.8 \mathrm{~Hz}$ ), 3.78 (s, 3H), 3.56 (s, 3H). ${ }^{13} \mathrm{C}$ NMR $(100.59 \mathrm{MHz}$, $\mathrm{CDCl}_{3}$ ) 168.0, 167.7, 138.5, 137.3, 133.0, 129.4, 128.8, 117.1, 57.2, 52.7, 52.6, 49.0. MS (EI) 223 (100), 191 (17), 163 (23), 151 (85), 128 (19), 115 (53), 59 (15). $[\alpha]_{D}^{22}=+40.9\left(\mathrm{c} 1.00, \mathrm{CHCl}_{3}\right)$ for $97 \%$ ee. Absolute configuration was determined by comparison with published data. ${ }^{3}$ Ee was measured by chiral SFC analysis with a Regis (R,R) WHELK-O column ( $2 \% \mathrm{MeOH}$ during $2 \mathrm{~min}$, then $1 \% \mathrm{~min}^{-1}$, flow rate $2 \mathrm{~mL} / \mathrm{min}$, pressure 130 bars, $\left.30^{\circ} \mathrm{C}\right) \mathrm{R}_{\mathrm{T}}: 3.30(+), 3.82(-)$.
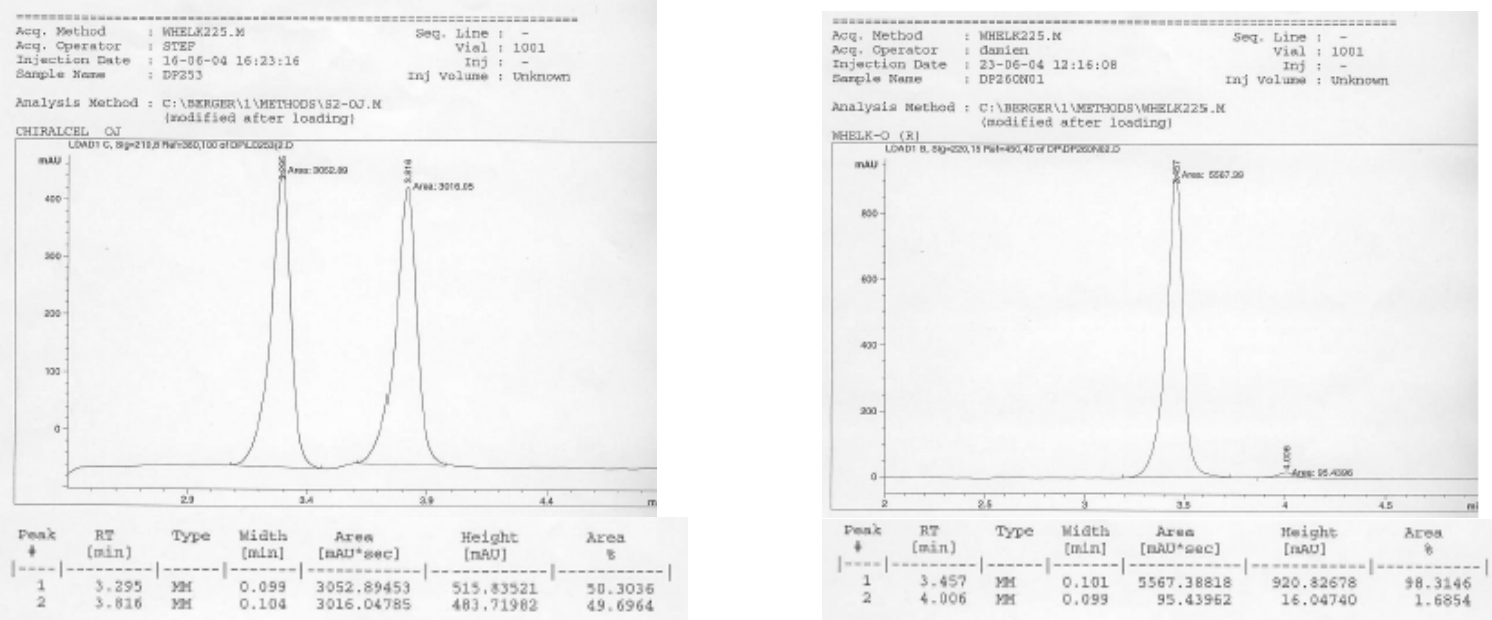<smiles>C=CC(C(=O)OC)C(C(=O)OC)c1ccc(OC)cc1</smiles>

(+)-2-[1-(4-Methoxy-phenyl)-allyl]-malonic acid dimethyl ester $2 \mathbf{f}^{5}{ }^{1} \mathrm{H}$ NMR (400.13 MHz, $\left.\mathrm{CDCl}_{3}\right) 7.14(\mathrm{~d}, 2 \mathrm{H}, J=8.6 \mathrm{~Hz}), 6.85$ (d, $2 \mathrm{H}, J=8.6 \mathrm{~Hz}$ ), 5.97 (ddd, $1 \mathrm{H}, J=17.1,10.1,7.8 \mathrm{~Hz}$ ), 5.09 (d, $1 \mathrm{H}, J=15.4 \mathrm{~Hz}$ ), 5.06 (d, $1 \mathrm{H}, J=8.6 \mathrm{~Hz}), 4.06$ (dd, $1 \mathrm{H}, J=10.9$, $8.3 \mathrm{~Hz}$ ), 3.82 (d, $1 \mathrm{H}, J=10.9 \mathrm{~Hz}), 3.77$ (s, 3H), 3.73 (s, 3H), 3.50 (s, 3H). ${ }^{13} \mathrm{C}$ NMR (100.59 MHz, $\left.\mathrm{CDCl}_{3}\right)$ 168.3, 167.9, 158.6, 138.1, 131.9, 129.0, 116.3, 114.0, 57.5, 55.2, 52.5, 48.9. MS (EI) 278 (14), 219 (35), 147 (100), 115 (13), 91 (16). $[\alpha]_{D}^{22}=+23.3$ (c 1.10, $\mathrm{CHCl}_{3}$ ) for $97 \%$ ee. Ee was measured by chiral SFC analysis with a Regis (R,R) WHELK-O column (2\% MeOH during $2 \mathrm{~min}$, then $1 \% \mathrm{~min}^{-1}$, flow rate $2 \mathrm{~mL} / \mathrm{min}$, pressure 130 bars, $\left.30^{\circ} \mathrm{C}\right) \mathrm{R}_{\mathrm{T}}$ : $4.99(+), 5.61(-)$.
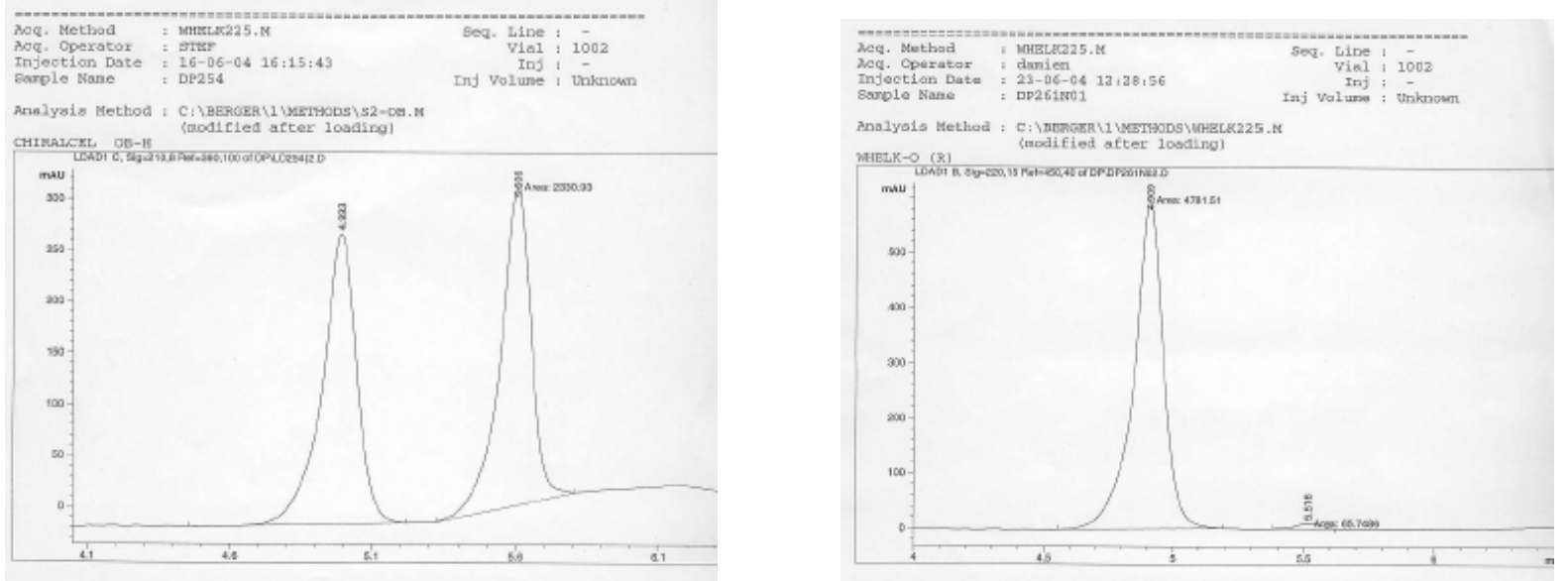

\footnotetext{
${ }^{5}$ You, S.-L.; Zhu, X.-Z.; Luo, Y.-M.; Hou, X.-L.; Dai, L.-X. J. Am. Chem. Soc. 2001, 123, 7471-7472.
} 

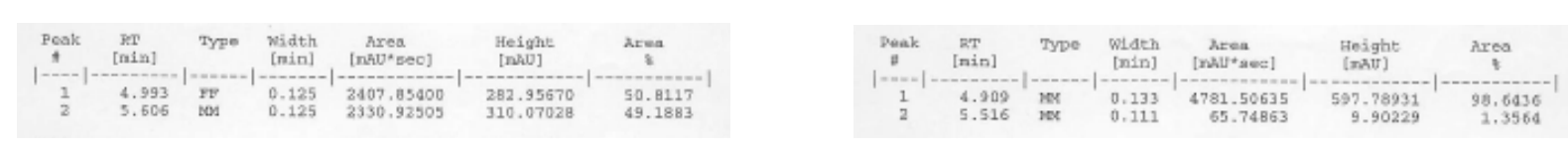<smiles>C=CC(C(=O)OC)C(C(=O)OC)c1ccccc1OC</smiles>

(+)-2-[1-(2-Methoxy-phenyl)-allyl]-malonic acid dimethyl ester $2 \mathbf{~ g ~}^{\mathbf{1}} \mathrm{H}$ NMR (400.13 MHz, CDCl 3 ) 7.20 (td, $1 \mathrm{H}, J=7.6,1.8 \mathrm{~Hz}$ ), 7.16 (dd, $1 \mathrm{H}$, $J=7.6,1.5 \mathrm{~Hz}$ ), 6.90-6.84 (m, 2H), 6.14 (ddd, $1 \mathrm{H}, J=17.0,10.1,8.6$ $\mathrm{Hz}$ ), 5.12 (dt, $1 \mathrm{H}, J=17.2,1.3 \mathrm{~Hz}$ ), 5.04 (dd, $1 \mathrm{H}, J=10.1,0.8 \mathrm{~Hz}$ ), 4.33 (dd, $1 \mathrm{H}, J=10.6,8.6 \mathrm{~Hz}$ ), 4.18 (d, $1 \mathrm{H}, J=10.6 \mathrm{~Hz}$ ), 3.85 (s, 3H), 3.72 (s, 3H), 3.49 (s, 3H). ${ }^{13} \mathrm{C}$ NMR (100.59 $\left.\mathrm{MHz}, \mathrm{CDCl}_{3}\right)$ 168.7, 168.3, 157.1, 136.9, 129.5, 128.3, 128.1, 120.7, 116.8, 111.1, 55.5, 55.4, 52.4, 52.3, 46.2. MS (EI) 278 (10), 219 (53), 187 (16), 159 (12), 147 (100), 131 (11), 115 (17), 91 (26). Anal Calcd for $\mathrm{C}_{15} \mathrm{H}_{18} \mathrm{O}_{5}$ : C, 64.74; H, 6.52; O, 28.74. Found: C, 64.46; H, 6.51; O, 29.03. $[\alpha]_{D}^{22}=+31.1(c$ $1.09, \mathrm{CHCl}_{3}$ ) for $79 \%$ ee. Ee was measured by chiral SFC analysis with a Regis $(\mathrm{R}, \mathrm{R})$ WHELK-O column ( $1 \% \mathrm{MeOH}$, flow rate $2 \mathrm{~mL} / \mathrm{min}$, pressure 130 bars, $\left.0^{\circ} \mathrm{C}\right) \mathrm{R}_{\mathrm{T}}: 5.67(+)$, $6.30(-)$.
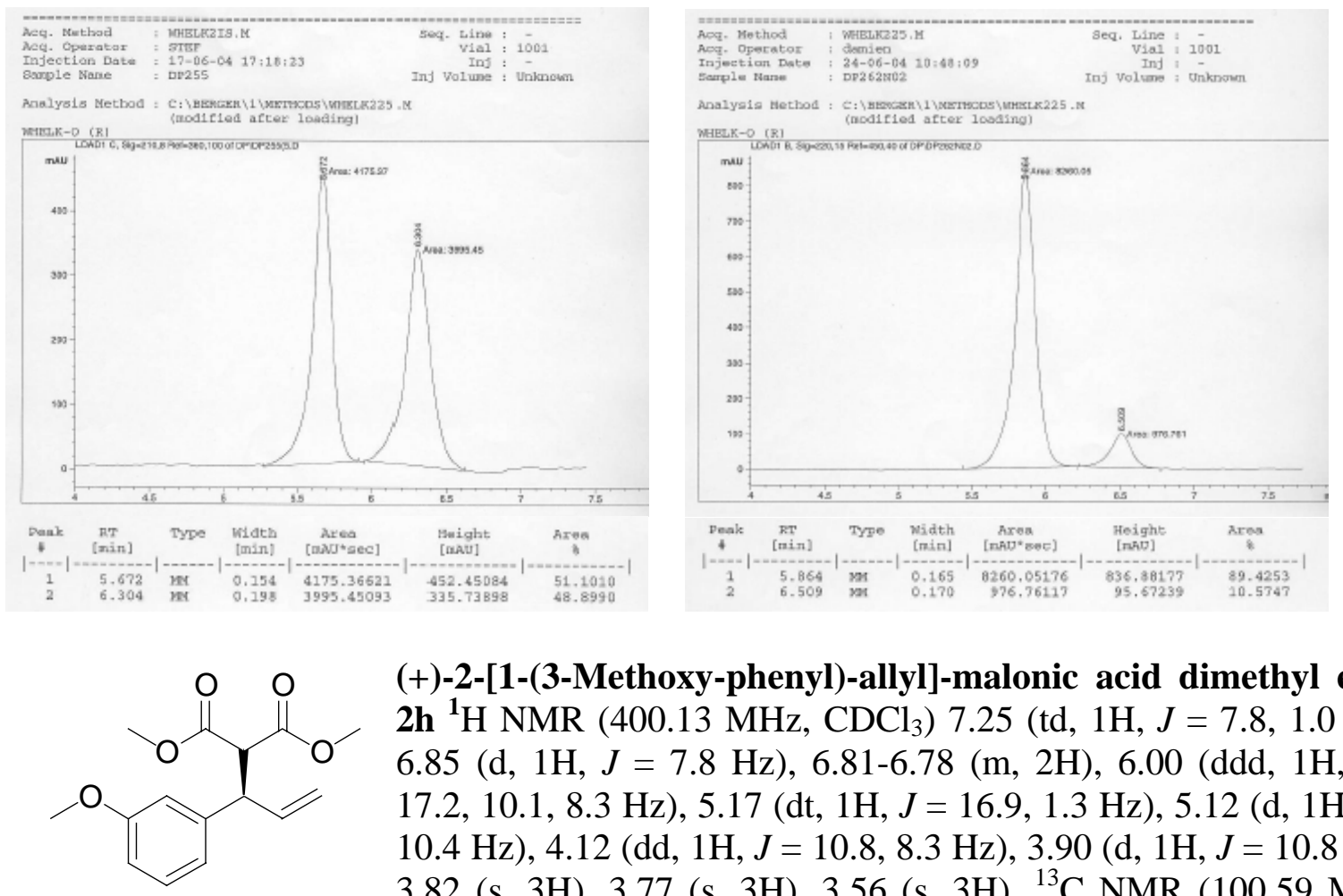

(+)-2-[1-(3-Methoxy-phenyl)-allyl]-malonic acid dimethyl ester 2h ${ }^{1} \mathrm{H}$ NMR (400.13 MHz, $\left.\mathrm{CDCl}_{3}\right) 7.25$ (td, $1 \mathrm{H}, J=7.8,1.0 \mathrm{~Hz}$ ), $6.85(\mathrm{~d}, 1 \mathrm{H}, J=7.8 \mathrm{~Hz}), 6.81-6.78(\mathrm{~m}, 2 \mathrm{H}), 6.00$ (ddd, $1 \mathrm{H}, J=$ 17.2, 10.1, $8.3 \mathrm{~Hz}$ ), 5.17 (dt, $1 \mathrm{H}, J=16.9,1.3 \mathrm{~Hz}$ ), 5.12 (d, $1 \mathrm{H}, J=$ $10.4 \mathrm{~Hz}$ ), 4.12 (dd, 1H, $J=10.8,8.3 \mathrm{~Hz}$ ), 3.90 (d, $1 \mathrm{H}, J=10.8 \mathrm{~Hz}$ ), $3.82(\mathrm{~s}, 3 \mathrm{H}), 3.77(\mathrm{~s}, 3 \mathrm{H}), 3.56(\mathrm{~s}, 3 \mathrm{H}) .{ }^{13} \mathrm{C}$ NMR $(100.59 \mathrm{MHz}$, $\left.\mathrm{CDCl}_{3}\right)$ 168.2, 159.7, 141.6, 137.7, 129.7, 120.1, 116.7, 113.8, 112.4, 57.3, 55.2, 52.6, 52.5, 49.8. MS (EI) 278 (28), 219 (100), 203 (10), 187 (38), 173 (10), 159 (40), 147 (86), 132 (12), 115 (34), 91 (35), 77 (13), 59 (12). Anal Calcd for $\mathrm{C}_{15} \mathrm{H}_{18} \mathrm{O}_{5}$ : C, 64.74; H, 6.52; O, 28.74. Found: C, 64.49; H, 6.48; O, 29.03. $[\alpha]_{D}^{22}=+28.9\left(\right.$ c 1.08, $\left.\mathrm{CHCl}_{3}\right)$ for $96 \%$ ee. Ee was measured by chiral SFC analysis with a Regis (R,R) WHELK-O column (1\% MeOH, flow rate $2 \mathrm{~mL} / \mathrm{min}$, pressure 130 bars, $\left.0^{\circ} \mathrm{C}\right) \mathrm{R}_{\mathrm{T}}$ : $5.95(+), 6.54(-)$. 

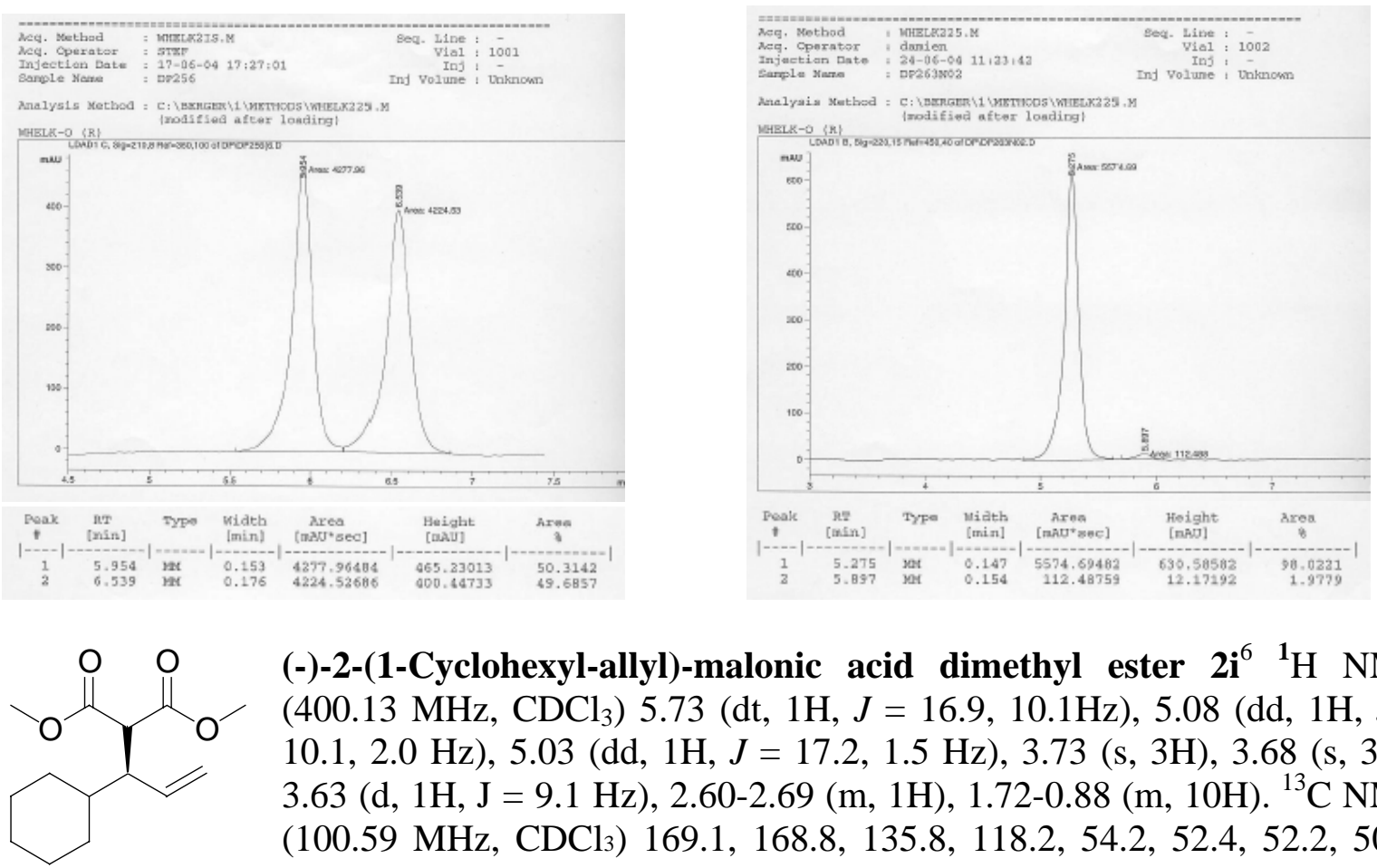

(-)-2-(1-Cyclohexyl-allyl)-malonic acid dimethyl ester $2 \mathbf{i}^{6}{ }^{\mathbf{1}} \mathrm{H} \quad \mathrm{NMR}$ $\left(400.13 \mathrm{MHz}, \mathrm{CDCl}_{3}\right) 5.73(\mathrm{dt}, 1 \mathrm{H}, J=16.9,10.1 \mathrm{~Hz}), 5.08$ (dd, $1 \mathrm{H}, J=$ 10.1, $2.0 \mathrm{~Hz}$ ), 5.03 (dd, $1 \mathrm{H}, J=17.2,1.5 \mathrm{~Hz}), 3.73$ (s, 3H), 3.68 (s, 3H), $3.63(\mathrm{~d}, 1 \mathrm{H}, \mathrm{J}=9.1 \mathrm{~Hz}), 2.60-2.69(\mathrm{~m}, 1 \mathrm{H}), 1.72-0.88(\mathrm{~m}, 10 \mathrm{H}) .{ }^{13} \mathrm{C} \mathrm{NMR}$ (100.59 MHz, $\left.\mathrm{CDCl}_{3}\right)$ 169.1, 168.8, 135.8, 118.2, 54.2, 52.4, 52.2, 50.1, 39.1, 28.6, 26.3. MS (EI) 195 (11), 172 (20), 140 (11), 133 (14), 122 (100), 113 (72), 107 (29), 101 (20), 93 (26), 81 (90), 77 (13), 67 (52) , 59 (31), 55 (75). It was impossible to get rid of $6 \%$ of starting material. $[\alpha]_{D}^{22}=-0.9\left(c 0.98, \mathrm{CHCl}_{3}\right)$ for $98 \%$ ee and $6 \%$ starting material. Ee was measured by chiral GC analysis with a Chiraldex G-TA column (80-0-1-170-5) $\mathrm{R}_{\mathrm{T}}$ : 44.67 (-), $45.00(+)$.
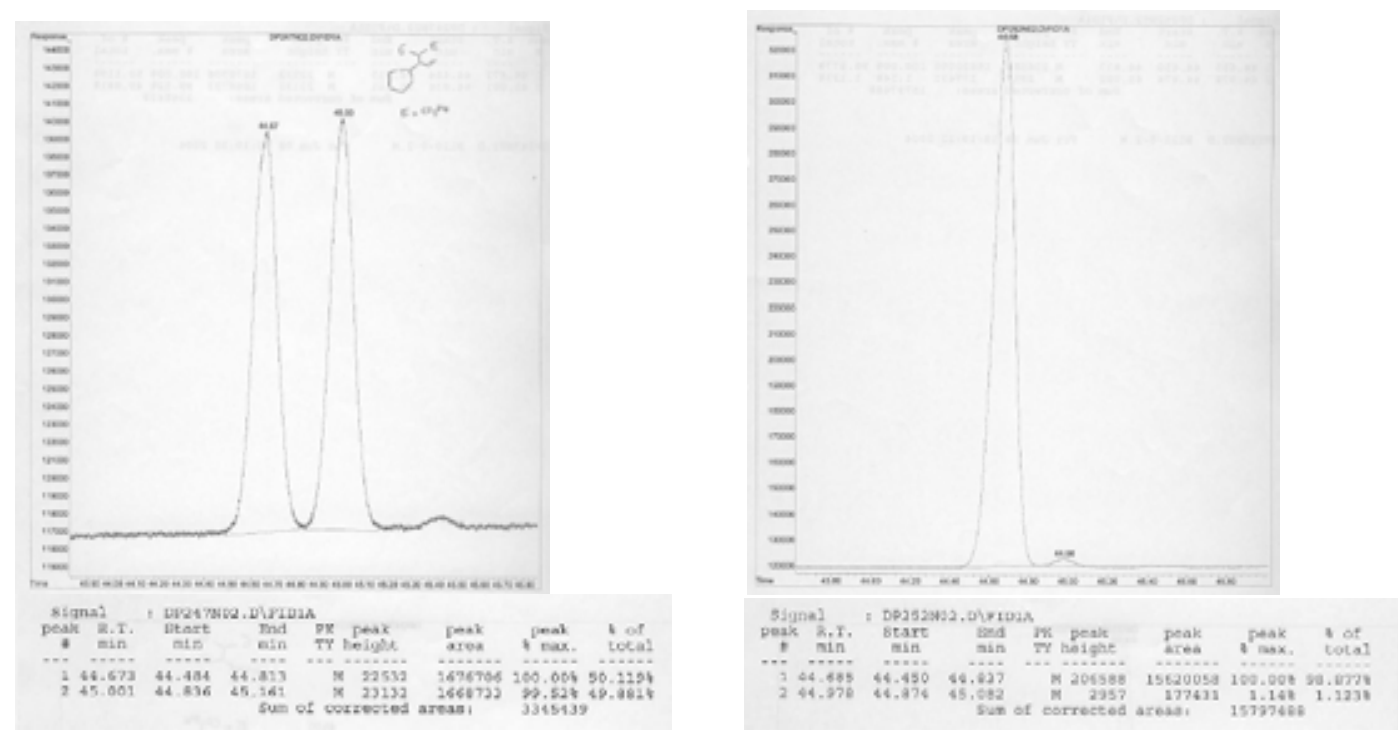<smiles>C=CC(CCC)C(C(=O)OC)C(=O)OC</smiles>

(+)-2-(1-Propyl-allyl)-malonic acid dimethyl ester $\mathbf{2 j}^{\mathbf{7}} \mathrm{H}$ NMR (400.13 $\left.\mathrm{MHz} \mathrm{CDCl}_{3}\right) 5.66(\mathrm{dt}, 1 \mathrm{H}, J=16.9,10.1 \mathrm{~Hz}), 5.11(\mathrm{~m}, 2 \mathrm{H}), 3.76(\mathrm{~s}, 3 \mathrm{H})$, 3.71 (s, 3H), 3.40 (d, $1 \mathrm{H}, \mathrm{J}=9.1 \mathrm{~Hz}), 2.79$ (qd, $1 \mathrm{H}, J=9.3,3.0 \mathrm{~Hz}), 1.45-$ $1.21(\mathrm{~m}, 4 \mathrm{H}), 0.91(\mathrm{t}, 3 \mathrm{H}, J=6.8 \mathrm{~Hz}) .{ }^{13} \mathrm{C} \mathrm{NMR}\left(100.59 \mathrm{MHz}, \mathrm{CDCl}_{3}\right)$ 168.8, 168.6, 138.1, 117.4, 57.0, 52.4, 52.2, 44.1, 34.5, 20.2, 13.8. MS (EI) 171 (41), 155 (100), 151 (32), 139 (66), 132 (69), 126 (30), 123 (29), 113 (49), 100 (48), 97 (10), 95 (36),

\footnotetext{
${ }^{6}$ Evans, P. A.; Nelson, J. D. Tetrahedron Letters 1998, 39, 1725-1728.

${ }^{7}$ Takeuchi, R.; Kashio, M. J. Am. Chem. Soc. 1998, 120, 8647-8655.
} 
81 (50), 67 (41), 59 (51) , 55 (83). $[\alpha]_{D}^{22}=+2.3\left(\right.$ ( $1.12, \mathrm{CHCl}_{3}$ ) for $97 \%$ ee. Ee was measured by chiral GC analysis with a Chiraldex G-TA column (70-0-0.5-110-15-170) $\mathrm{R}_{\mathrm{T}}$ : $29.51(+), 30.41(-)$.
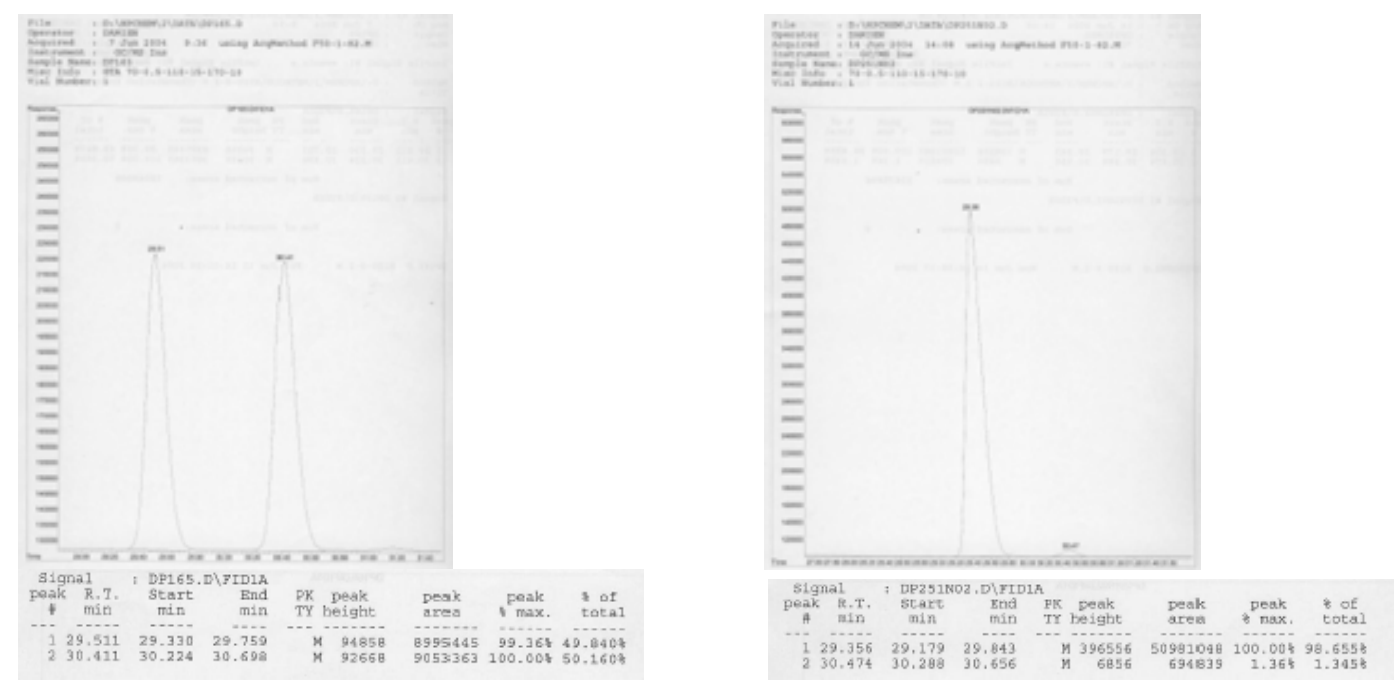


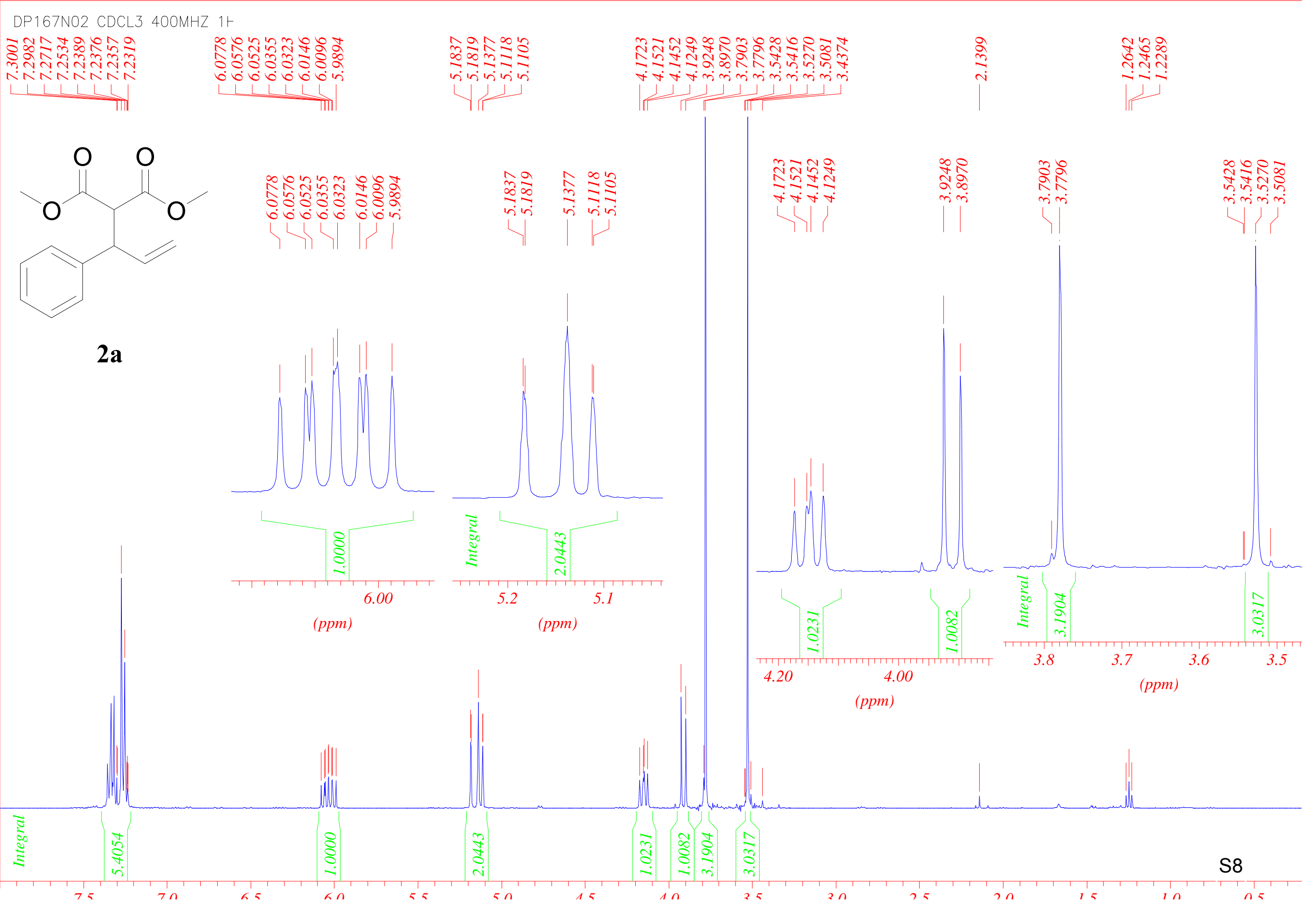




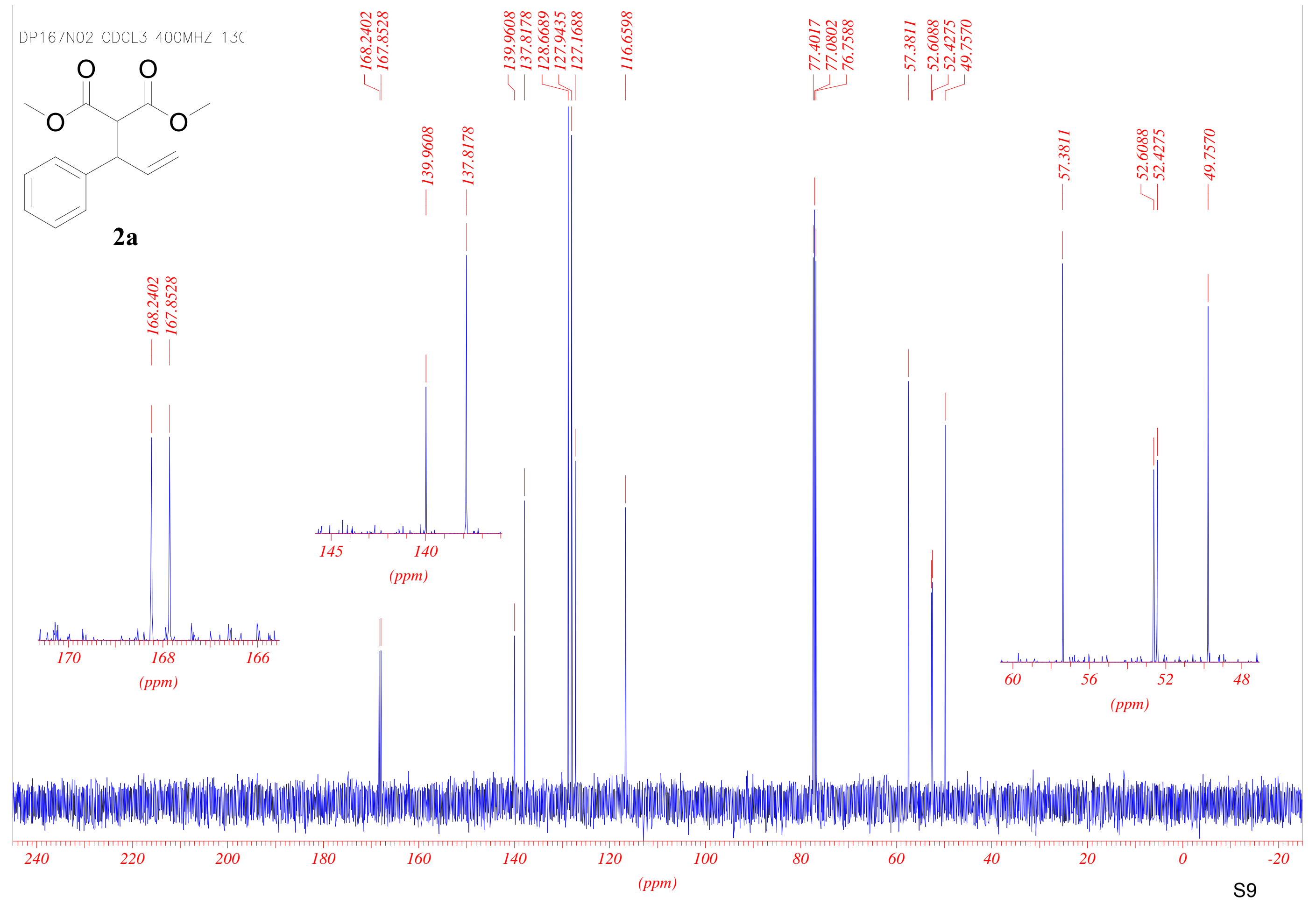




\section{DP241NO4 CDCL3 400MHZ 1F}

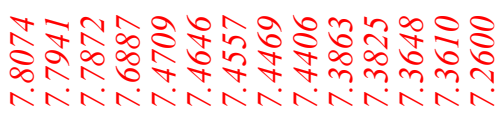

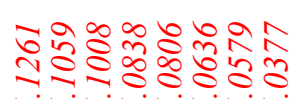

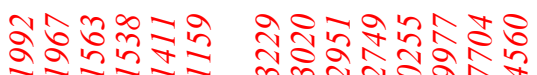

6.6 .6060

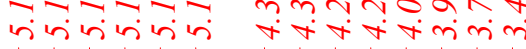

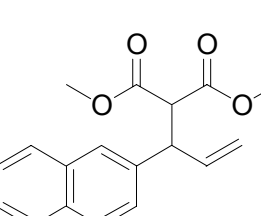

2b

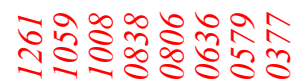

6.6 .6060 .6

nై

nn ñn
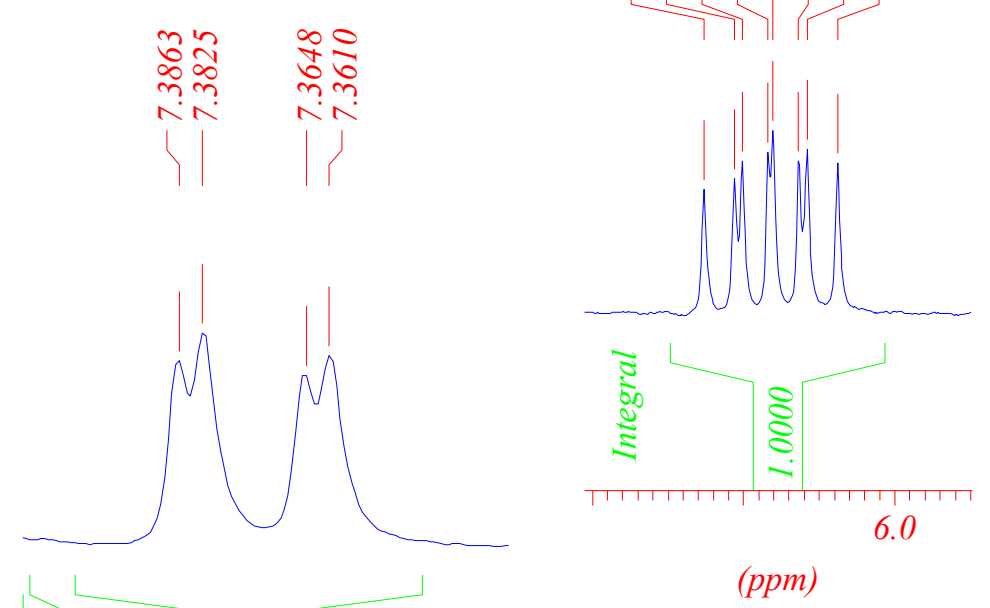

ลำ

in wivin

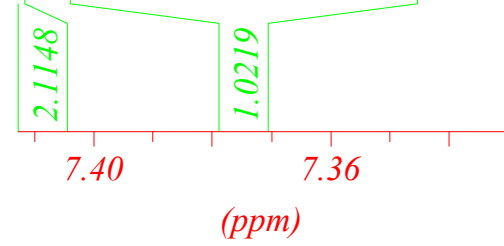

(ppm)

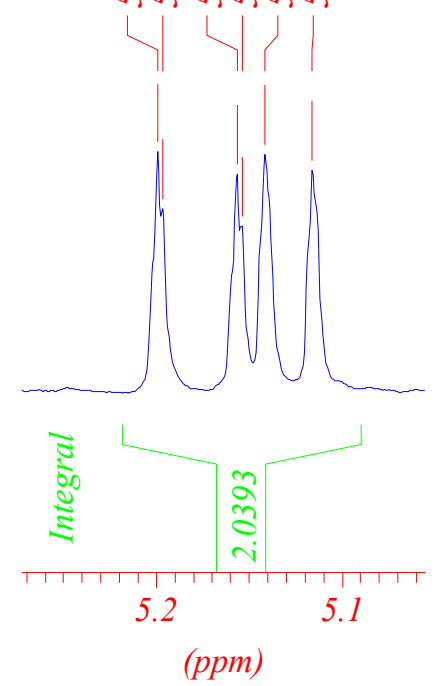

ป

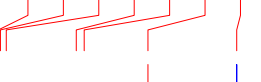




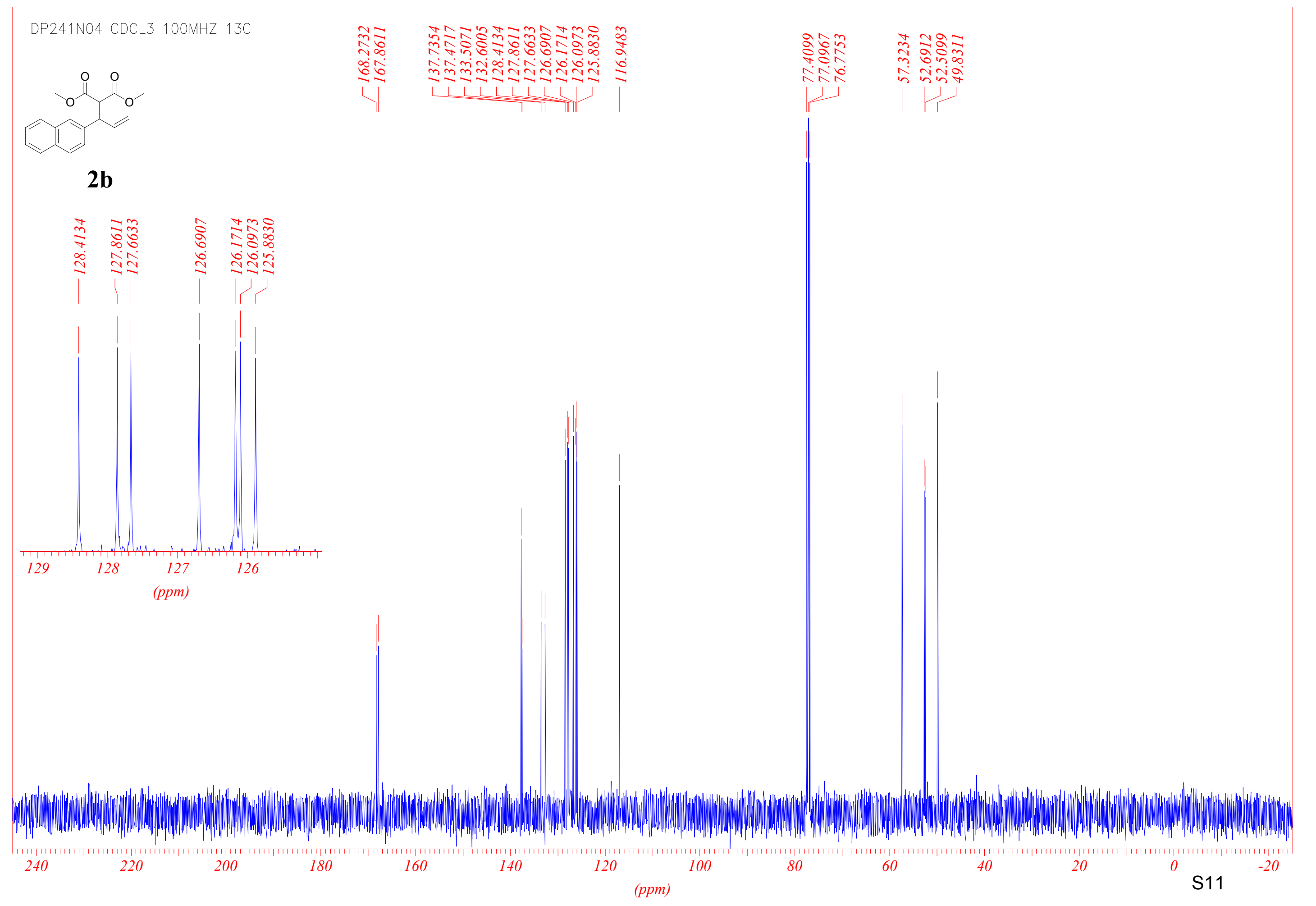




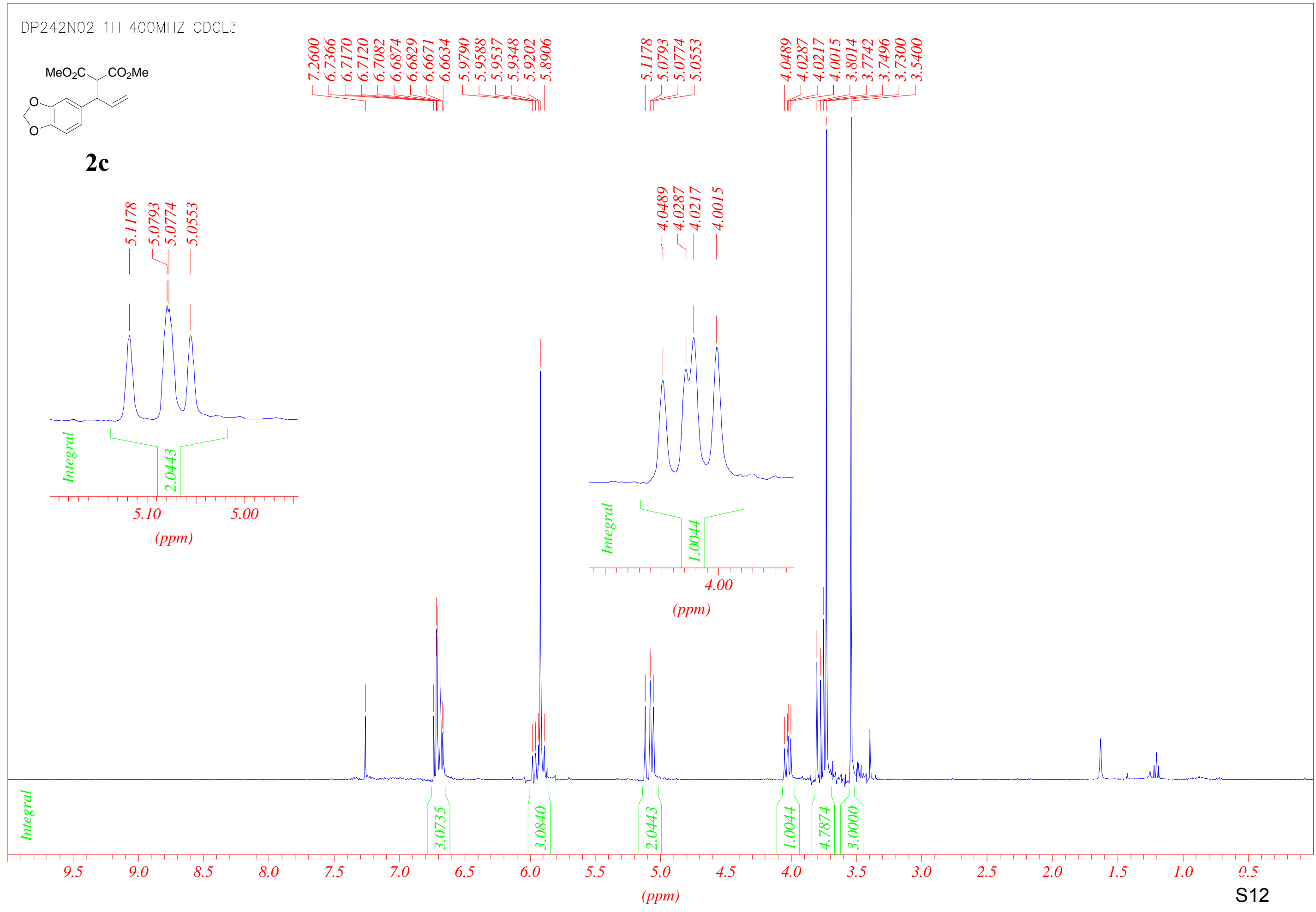




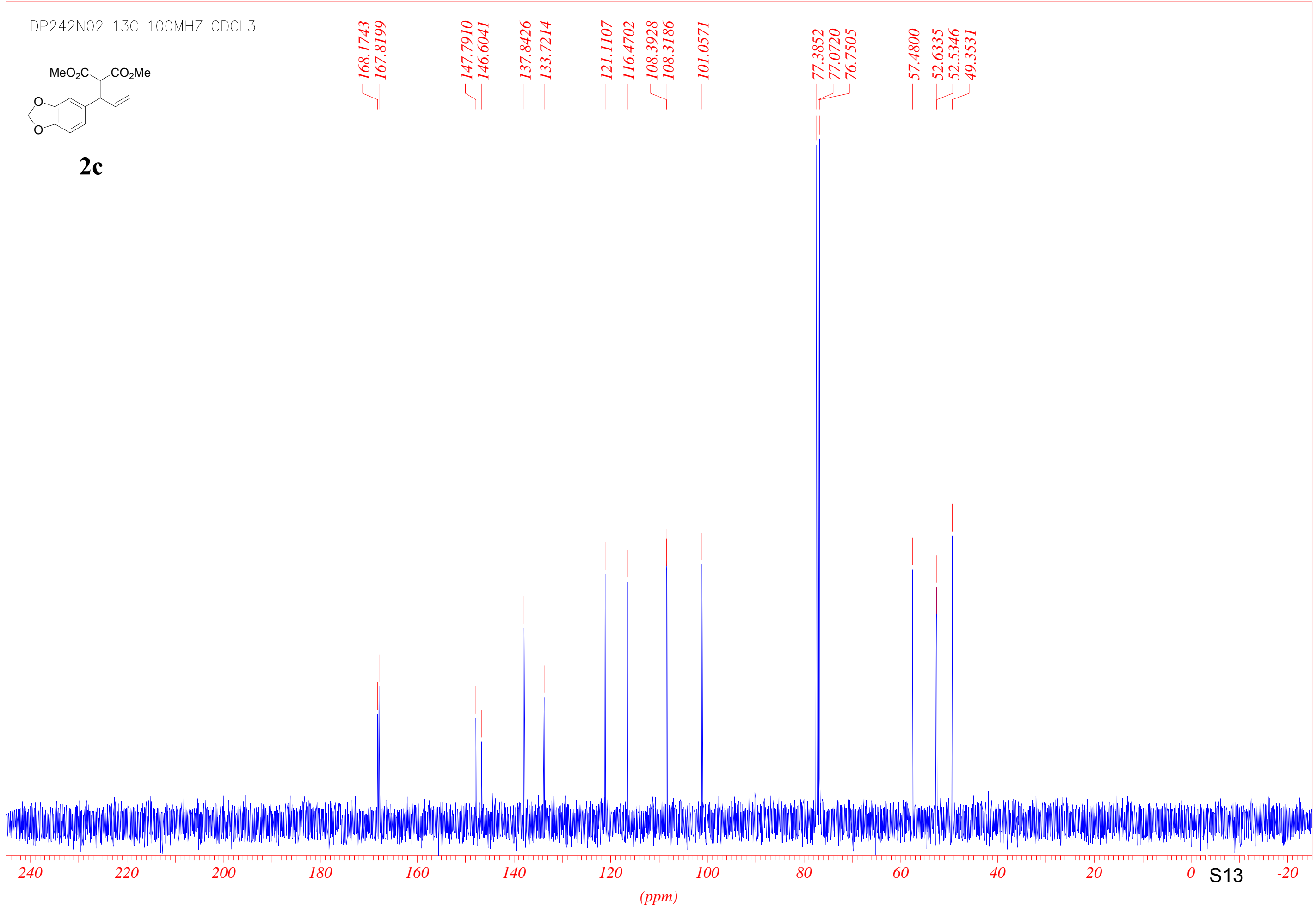




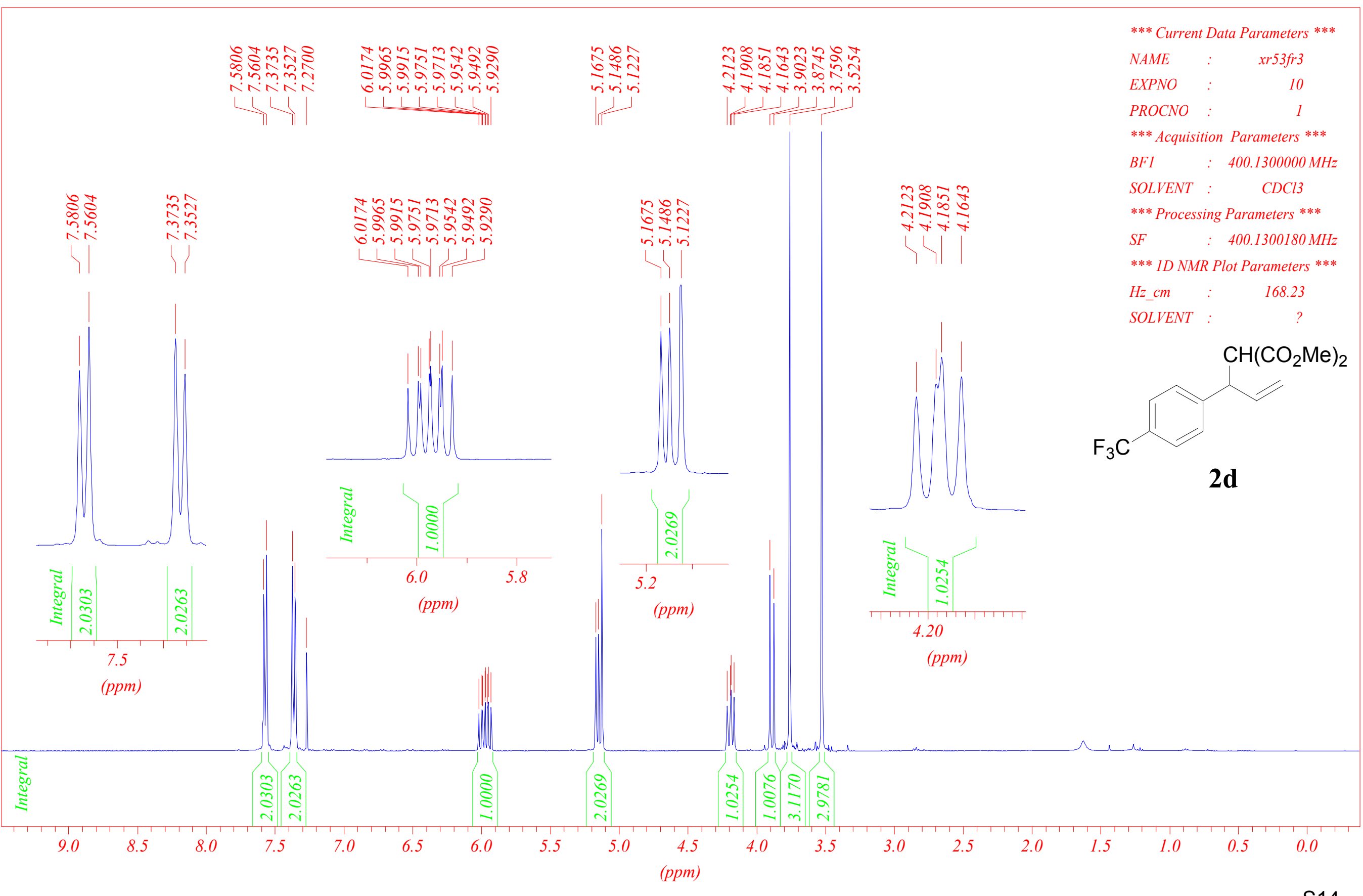




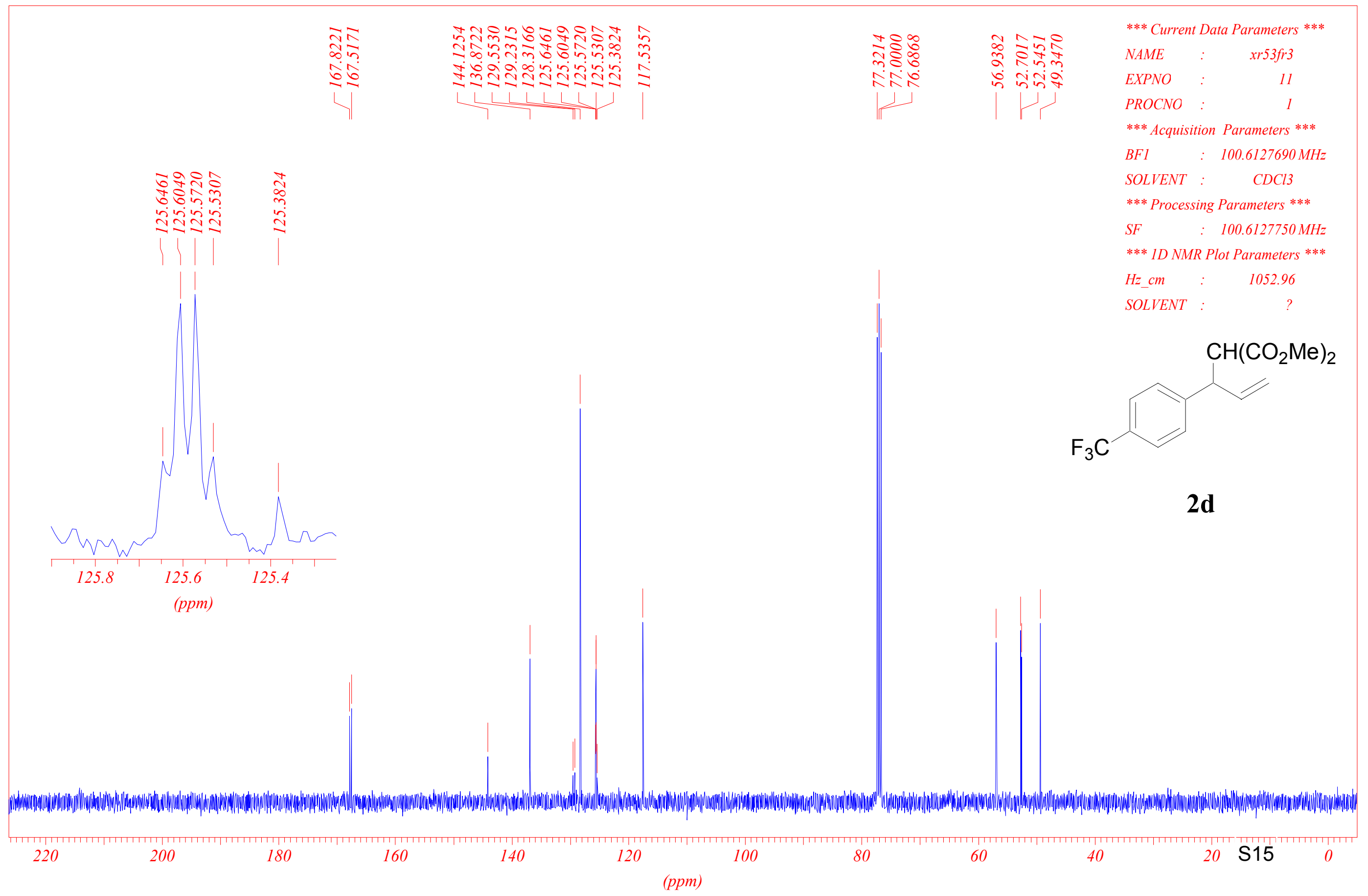




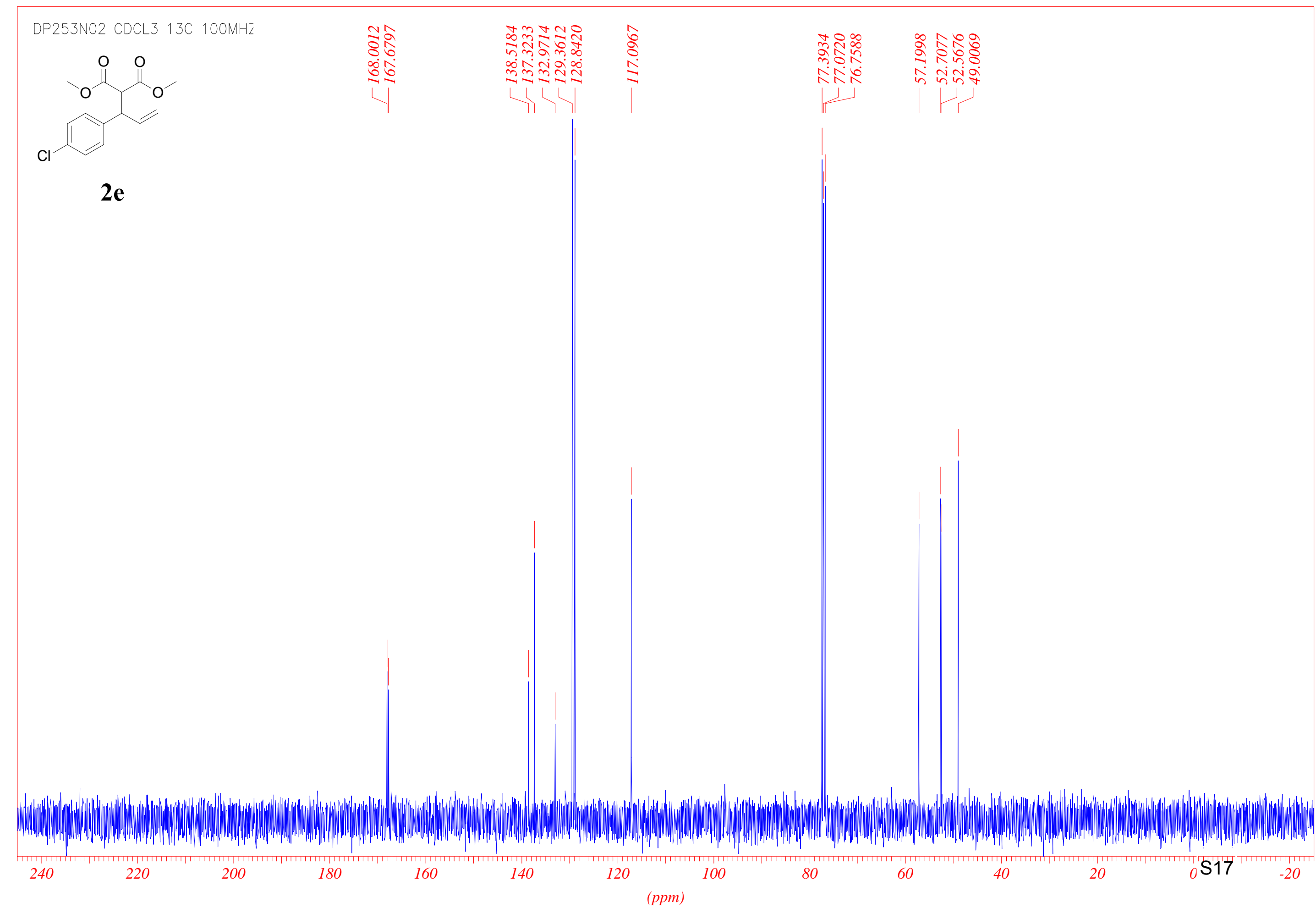




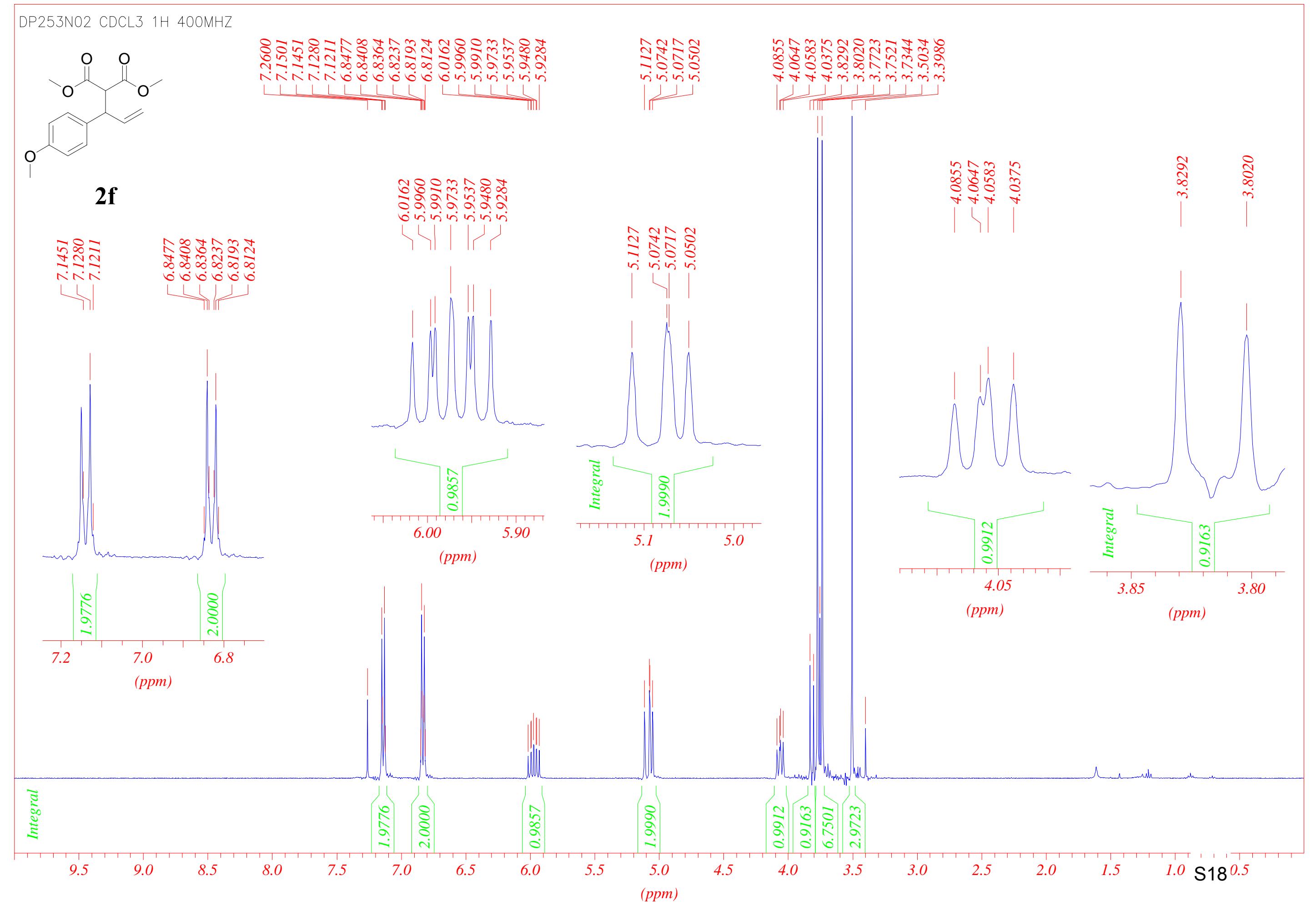


DP253N02 CDCL3 $13 \mathrm{C} \quad 100 \mathrm{MHZ}$

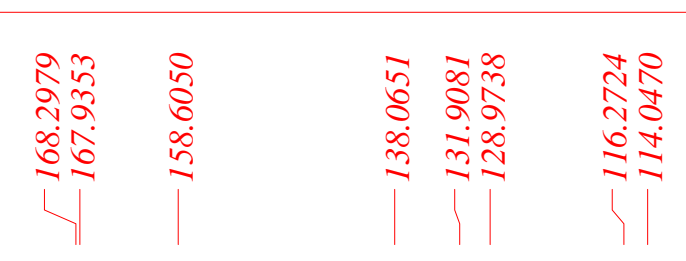

0

\section{2f}

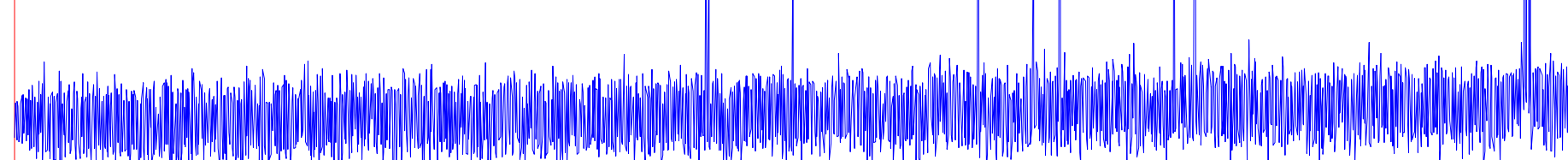

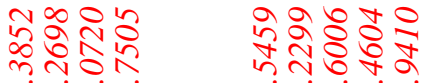

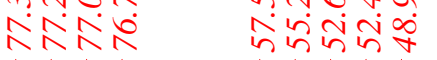




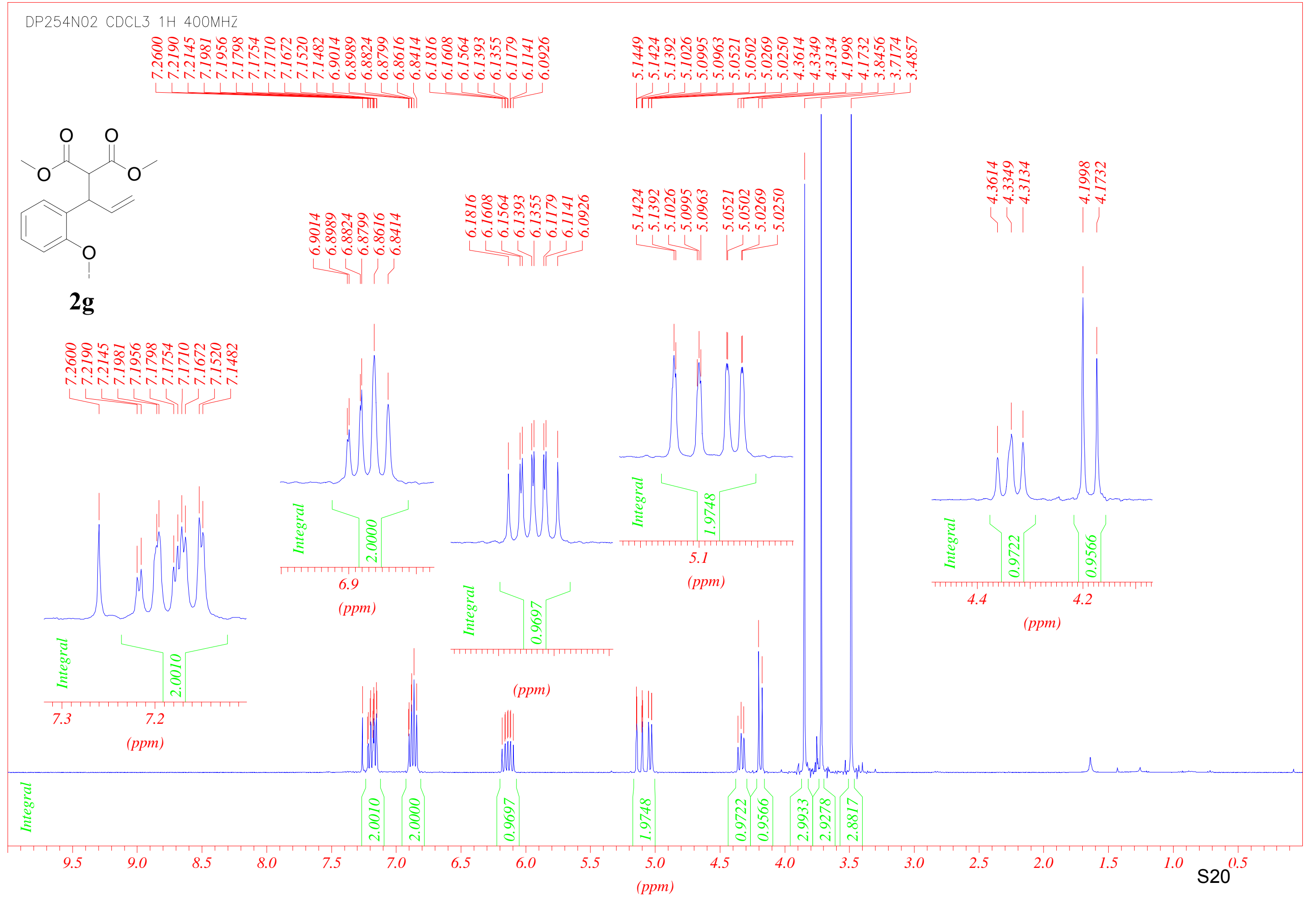




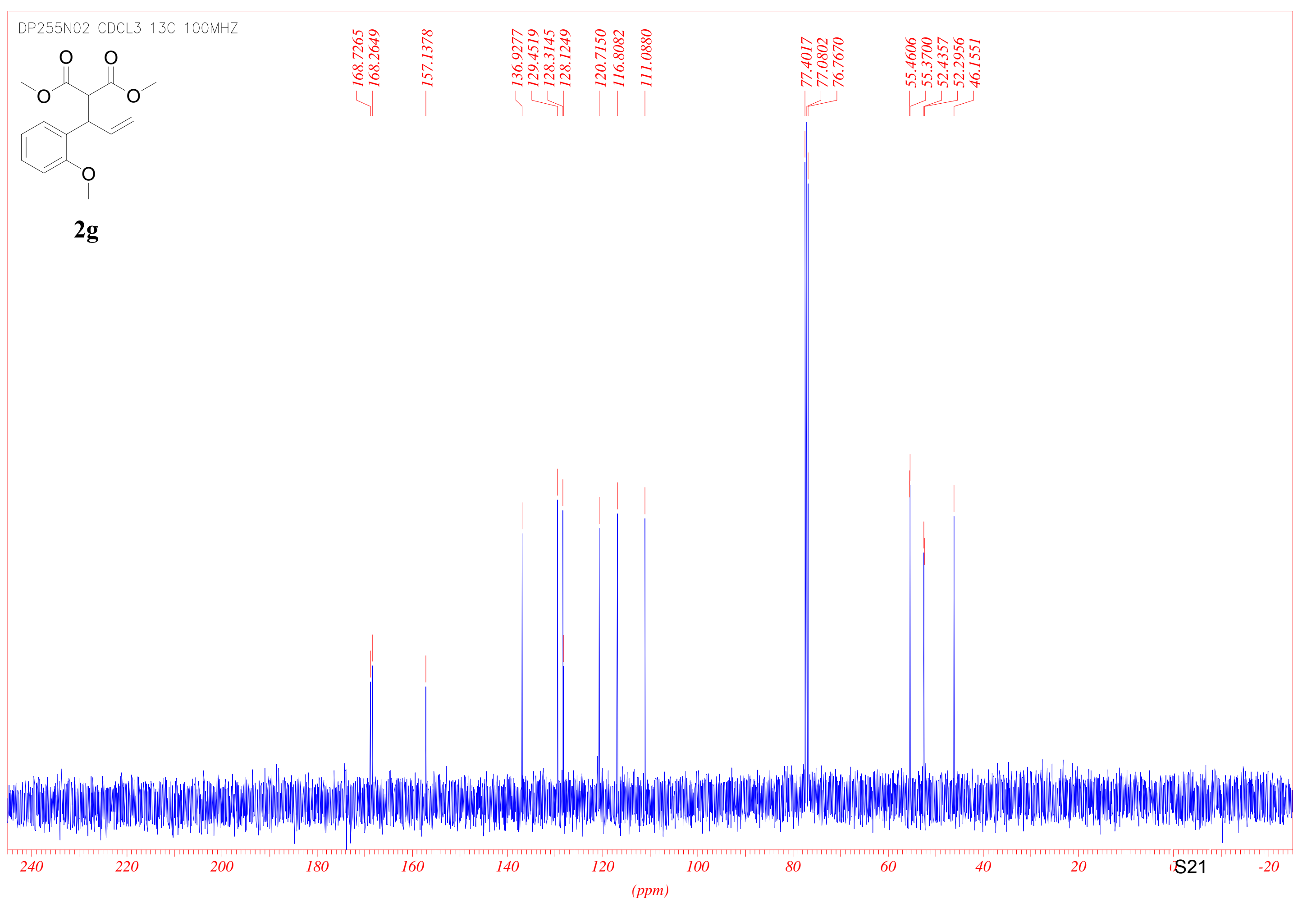




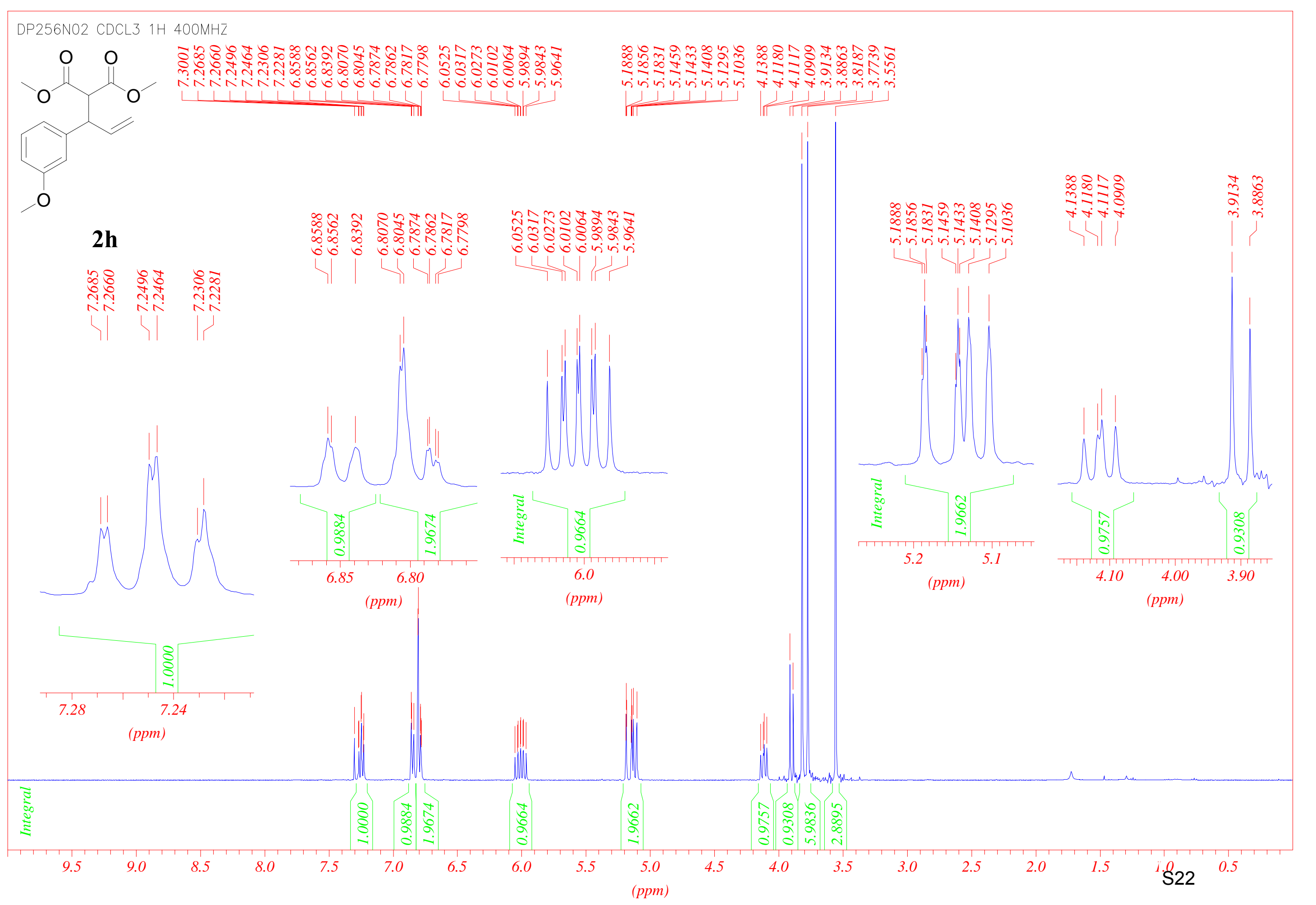




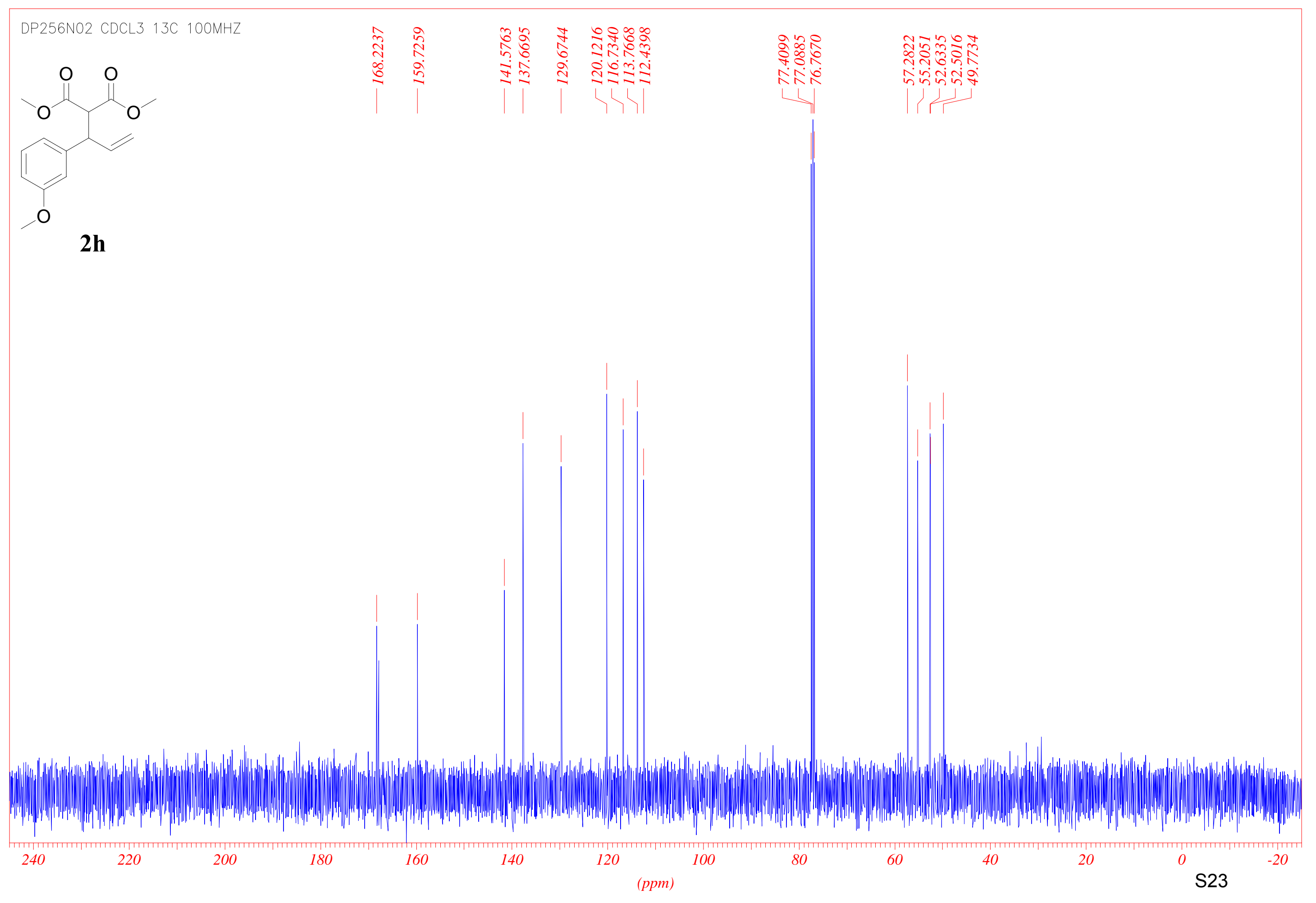




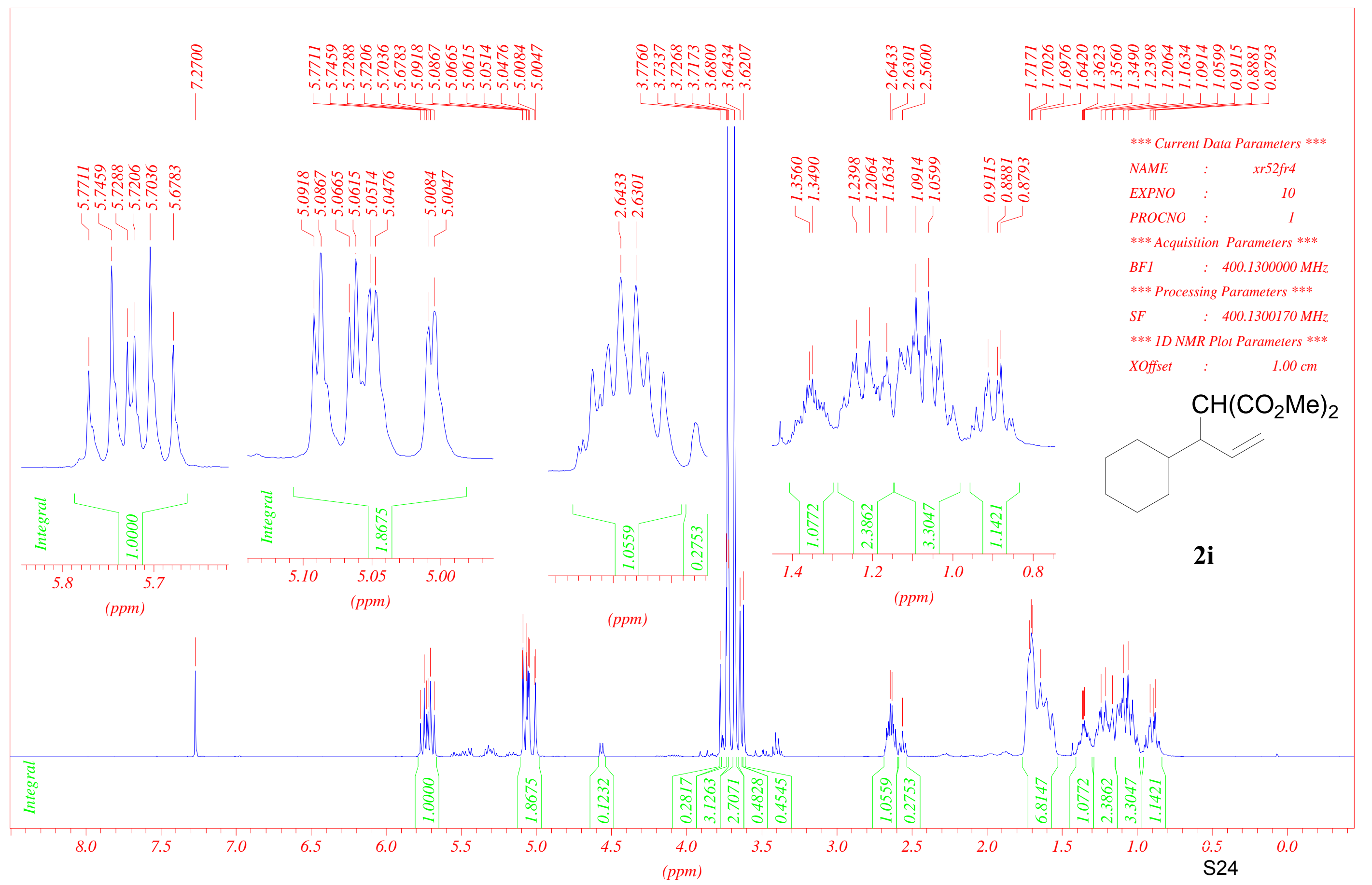




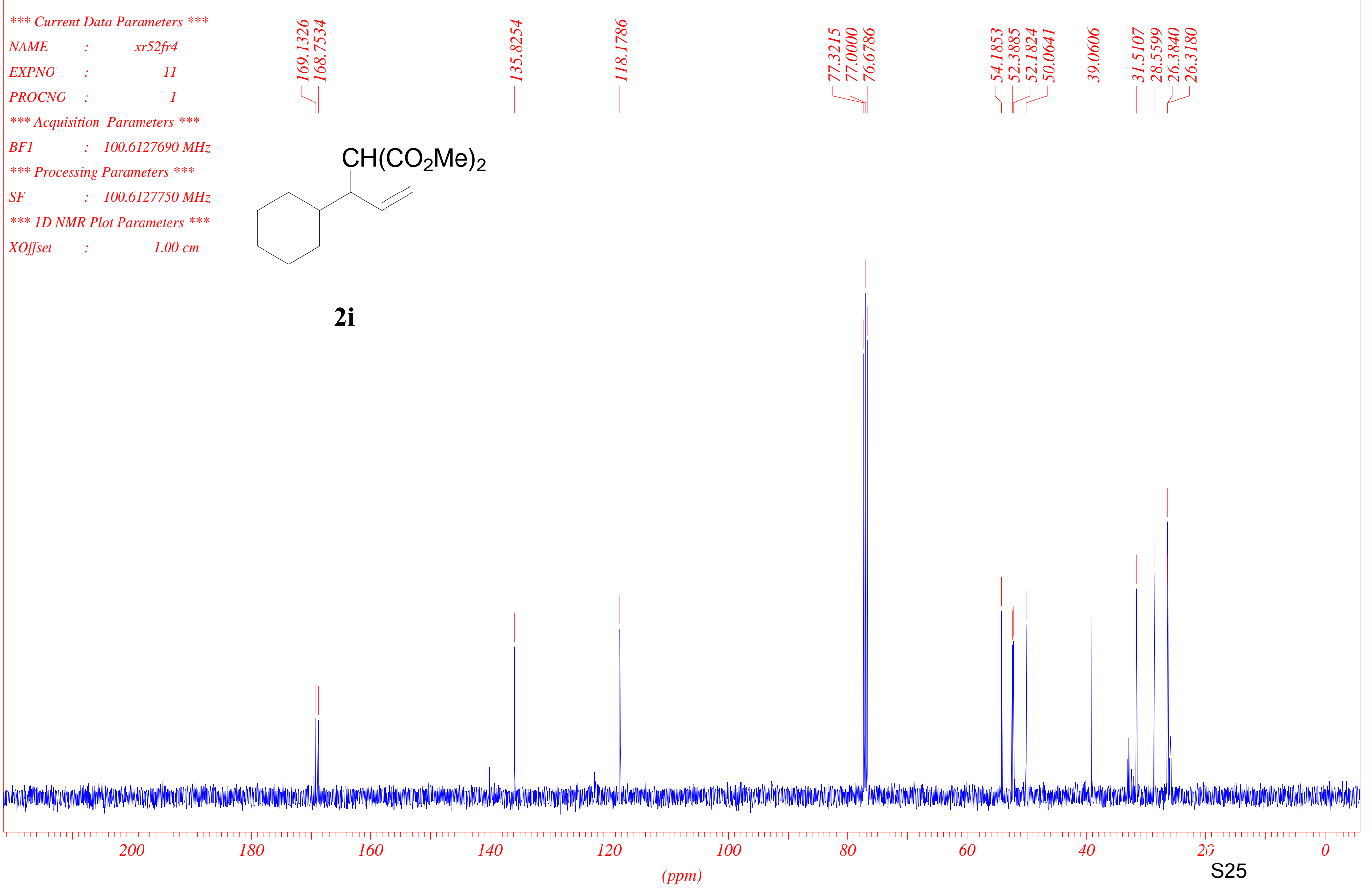




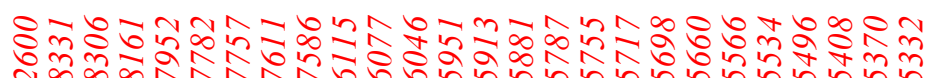

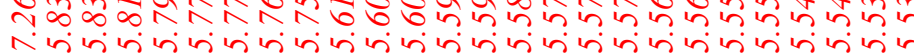

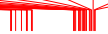

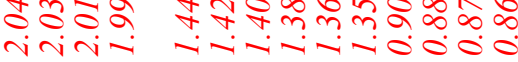

等

$2 \mathbf{j}$

\section{mై}

$\infty \infty$ रn

(l)

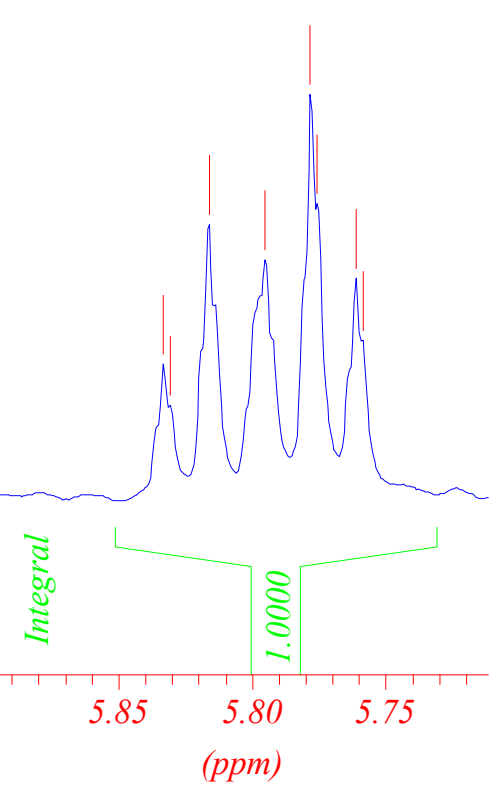

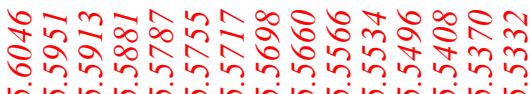

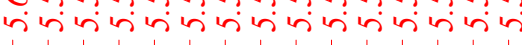
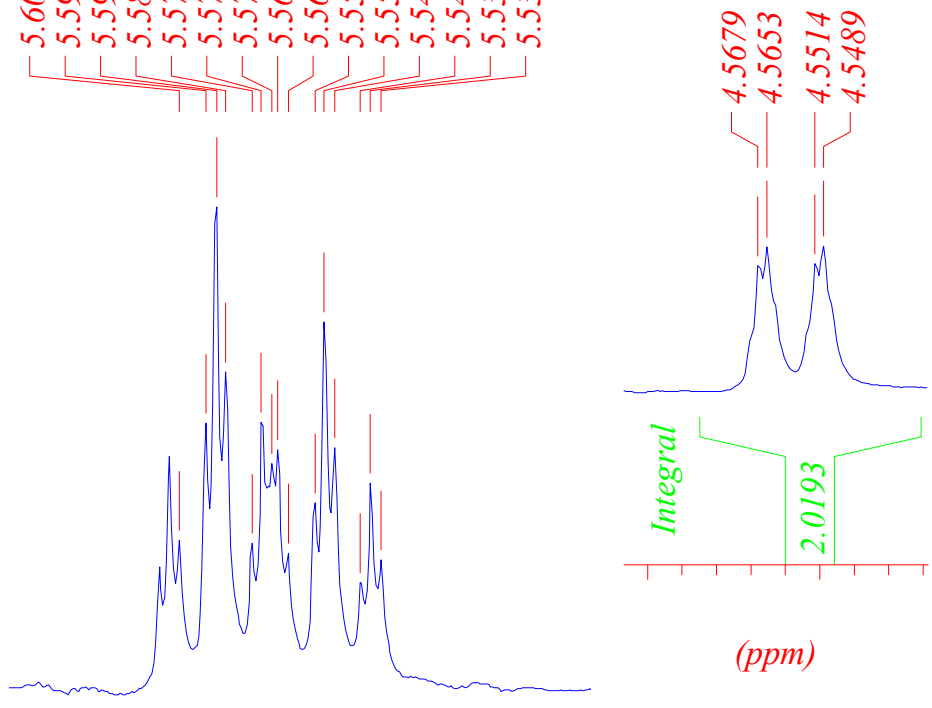

(ppm)

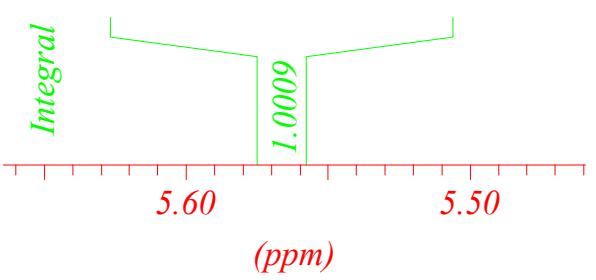

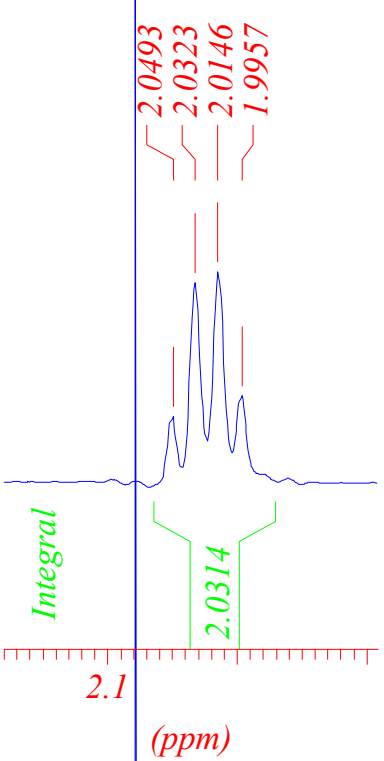

รัปำำล

i-i-i-i-n
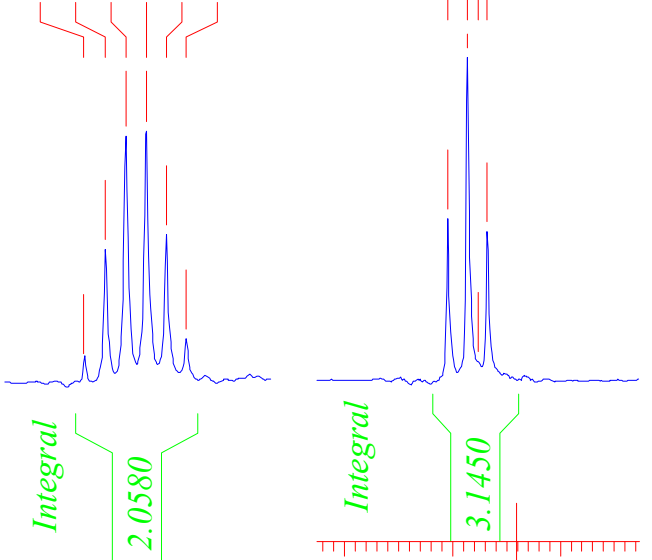

(ppm)

(рpm)

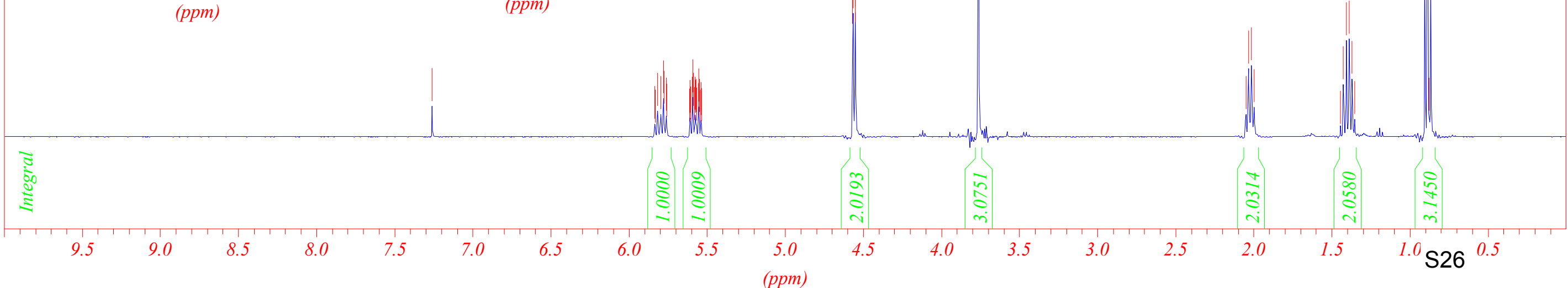


0

${ }^{\prime} 0^{\prime}$

\section{$\mathbf{2 j}$}

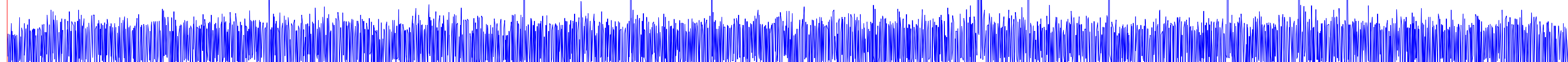

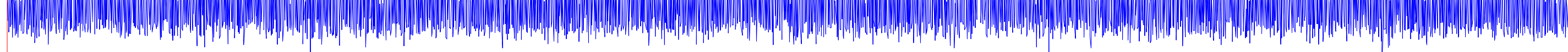

